\title{
Generating surfaces of daily meteorological variables over large regions of complex terrain
}

\author{
Peter E. Thornton*, Steven W. Running, Michael A. White \\ Numerical Terradynamics Simulation Group, School of Forestry, University of Montana, Missoula, \\ MT 59812, USA
}

\section{Abstract}

A method for generating daily surfaces of temperature, precipitation, humidity, and radiation over large regions of complex terrain is presented. Required inputs include digital elevation data and observations of maximum temperature, minimum temperature and precipitation from ground-based meteorological stations. Our method is based on the spatial convolution of a truncated Gaussian weighting filter with the set of station locations. Sensitivity to the typical heterogeneous distribution of stations in complex terrain is accomplished with an iterative station density algorithm. Spatially and temporally explicit empirical analyses of the relationships of temperature and precipitation to elevation were performed, and the characteristic spatial and temporal scales of these relationships were explored. A daily precipitation occurrence algorithm is introduced, as a precursor to the prediction of daily precipitation amount. Surfaces of humidity (vapor pressure deficit) are generated as a function of the predicted daily minimum temperature and the predicted daily average daylight temperature. Daily surfaces of incident solar radiation are generated as a function of Sun-slope geometry and interpolated diurnal temperature range. The application of these methods is demonstrated over an area of approximately $400000 \mathrm{~km}^{2}$ in the northwestern USA, for 1 year, including a detailed illustration of the parameterization process. A cross-validation analysis was performed, comparing predicted and observed daily and annual average values. Mean absolute errors (MAE) for predicted annual average maximum and minimum temperature were $0.7^{\circ} \mathrm{C}$ and $1.2^{\circ} \mathrm{C}$, with biases of $+0.1^{\circ} \mathrm{C}$ and $-0.1^{\circ} \mathrm{C}$, respectively. MAE for predicted annual total precipitation was $13.4 \mathrm{~cm}$, or, expressed as a percentage of the observed annual totals, $19.3 \%$. The success rate for predictions of daily precipitation occurrence was $83.3 \%$. Particular attention was given to the predicted and observed relationships between precipitation frequency and intensity, and they were shown to be similar. We tested the sensitivity of these methods to prediction grid-point spacing, and found that areal averages were unchanged for grids ranging in spacing from $500 \mathrm{~m}$ to $32 \mathrm{~km}$. We tested the dependence of the results on timestep, and found that the temperature prediction algorithms scale perfectly in this respect. Temporal scaling of precipitation predictions was complicated by the daily

* Corresponding author. 
occurrence predictions, but very nearly the same predictions were obtained at daily and annual timesteps. (C) 1997 Elsevier Science B.V.

\section{Introduction}

Research efforts in the hydrological and ecological sciences are increasingly being directed toward the application of knowledge gained at small spatial scales to questions framed over larger domains. Consequently, there is a growing need for a new collection of research tools and methods designed with attention to the particular needs and constraints of large-scale studies (Shuttleworth, 1988; Dolph and Marks, 1992; Troch et al., 1993). Reliable surface meteorological data are a basic requirement for hydrological and ecological research at any spatial scale, and is a particularly crucial component of studies of mass and energy transfer over large land surfaces. Our study of hydroecological processes at regional and continental scales has been hindered by the lack of a general method which meets the meteorological data requirements of such large-scale studies. Here we outline some of the basic requirements for meteorological data in studies of land-surface processes over large spatial domains, and we present methods by which these requirements can be met.

In contrast to plot-level research, for which on-site collection of pertinent near-surface meteorological data is straightforward and routine, large-scale studies of land-surface processes are often limited to the use of extant data sources. For studies over small domains it is possible to install and maintain the instruments necessary to gather all the required variables, but at some point, as the size of the spatial domain increases, this approach becomes prohibitively expensive. For such cases, the routine observation of meteorological variables across large station networks is a uniquely valuable source of information. Networks such as those operated in the USA by the National Weather Service (NWS Cooperative Observer Network) and by the Natural Resources Conservation Service (SNOTEL Network, USDA Soil Conservation Service, 1988) are of particular value for their wide geographic distribution, duration of record, frequency of observations, and standardized measurement techniques.

Only a limited selection of the meteorological variables relevant to studies of landsurface processes are routinely available from existing networks of surface stations. The majority of such stations record daily observations of temperature extrema (maximum temperature, TMAX, and minimum temperature, TMIN) and daily total precipitation (PRCP). Some stations record other variables, including wind speed, humidity, and various components of the surface radiation balance, but the spatial coverage of such data is limited. Previous studies (Running and Coughlan, 1988; McMurtrie et al., 1992) have defined the minimum required daily meteorological variables for accurate simulations of hydrological and ecological land-surface processes, as follows: precipitation, surface air temperature, surface air humidity, and incident shortwave radiation. A model, MTCLIM (Running et al., 1987; Hungerford et al., 1989; Glassy and Running, 1994), was developed to provide daily values for these variables in complex terrain, by extrapolating daily 
observations from one or sometimes two stations to a remote and uninstrumented site. MTCLIM generates the required variables from daily observations of TMAX, TMIN, and PRCP, given elevation, slope, aspect, latitude, and albedo at the observation and prediction sites. Extrapolation of temperatures with elevation is accomplished by user-specified lapse rates for TMAX and TMIN, assumed to apply throughout the year. Daily PRCP is extrapolated using a ratio of mean annual total precipitation between the sites of observation and prediction, with the predicted occurrence of precipitation events (wet days) duplicated from the observed time series of PRCP. Humidity is derived from an assumed relationship between TMIN and the dewpoint temperature. Incident shortwave radiation is derived from the diurnal temperature range and Sun-Earth geometry, after the methods of Bristow and Campbell (1984).

Studies ranging in spatial scale from point simulations (Running and Coughlan, 1988; Running, 1994), to single watershed simulations (Band et al., 1991, 1993; White and Running, 1994), to simulations over areas on the order of $1-2000 \mathrm{~km}^{2}$ (Running and Nemani, 1991, Nemani et al., 1993), have demonstrated the successful application of the basic MTCLIM logic. In these cases it has been assumed that observations from one or two nearby stations can reasonably represent the horizontal meteorological variability over the study region. For studies over progressively larger regions this assumption loses its validity. Whereas the original MTCLIM logic is concerned with extrapolation of meteorological data to a remote site, studies over large regions (greater than approximately $2000 \mathrm{~km}^{2}$ ) require an extension of this logic to include interpolations between an unspecified number of heterogeneously spaced observations in complex terrain. We present such an interpolation method here, and demonstrate its application in the context of the original MTCLIM logic.

A multiple-station logic allows an analysis of the spatial and temporal variation in the relationships of temperature and precipitation to elevation. The original MTCLIM used specified lapse rates for TMAX and TMIN, which were derived from regional observations and held constant in space and time. The relationship of PRCP to elevation assumed prior knowledge of the mean annual precipitation at both the observation and prediction sites, and no allowance was made for temporal variation. Here we incorporate methods which make use of a large number of observations at different elevations to objectively analyze the relationships of TMAX, TMIN, and PRCP to elevation. We also examine, in an empirical framework, the characteristic spatial and temporal scales of these relationships.

The parameterization, validation, and implementation of these methods are illustrated, employing a database of daily observations of TMAX, TMIN, and PRCP for 1 year (1989), for some 500 stations in the northwestern USA. The methods are implemented over an area of approximately $400000 \mathrm{~km}^{2}$. We examine the influence of prediction grid-point spacing on the areally averaged results. Finally, we examine the application of the same general methods over longer timesteps, in an effort to relate the results of daily predictions to predictions made in a more climatological mode.

Both the original MTCLIM and the extensions provided here are designed to generate sequences of daily meteorological variables which are as close as possible to actual daily sequences. Other methods exist which generate stochastic sequences of 
meteorological variables at daily, monthly, and other timesteps (e.g. Hutchinson, 1995; Wallis and Griffiths, 1995). Hutchinson (1995) has provided a general discussion of stochastic methods and their application in hydrological and ecological research.

\section{Background and model development}

Much of the recent literature concerning spatial interpolation of meteorological fields has focused on the generation of surfaces of long-term average or climatological precipitation. Particular attention has been given to the development of sophisticated statistical methods which, given certain assumptions, generate explicit optimality criteria and guarantees of unbiased predictions. Some examples are optimal interpolation (Gandin, 1965), kriging and its variants (e.g. Phillips et al., 1992), and smoothing splines (Hutchinson and Bischof, 1983). Simpler methods which lack such optimality criteria and guarantees of unbiasedness have been applied extensively for the determination of mean areal precipitation. The method of nearest neighbors (Thiessen, 1911) is an early example, and others include multiple nearest neighbors, inverse-distance weighting schemes, and arithmetic means. Several studies have compared various of these sophisticated and simple methods in the context of the areal distribution of rainfall (Creutin and Obled, 1982; Tabios and Salas, 1985; Phillips et al., 1992). Other studies have focused on one method but offered comparisons with others (Chua and Bras, 1982; Hevesi et al., 1992).

The comparison studies generate a somewhat surprising result: although the statistical methods are for the most part more accurate than the simple methods, they are not overwhelmingly so. For example, from Tabios and Salas (1985) (Table 10), there is no significant difference between inverse-distance methods and a suite of statistical methods based on coefficients of determination for estimates of mean annual precipitation from five sites in homogeneous terrain, whereas the nearest-neighbor method was significantly inferior, but only by approximately $10 \%$. Such results secure the hope that an effective, efficient interpolation method could be developed by borrowing elements from various simple methods and addressing the characteristics of those methods which appear to be most responsible for their relatively poor behavior in the comparison studies.

A recent example in this vein is the work of Daly et al. (1994), who have developed what can be considered a hybrid approach for distributing climatological precipitation, combining geographical and statistical elements, which they demonstrate to be both more flexible and more accurate than kriging and some of its variants. Another example is the method of climatologically aided interpolation (CAI) developed by Willmott and Robeson (1995) and applied to the interpolation of yearly temperature averages. CAI uses a relatively simple inverse-distance weighting scheme to adjust a spatially high-resolution climatology. The method produces low validation errors, and its accuracy is attributed in part to the incorporation of terrain effects provided by the high-resolution climatology. A third example, pertinent for its utilitarian and flexible approach, is the recursive filter objective analysis (RF) used in the operational analysis of meteorological satellite 
soundings (Hayden and Purser, 1995). RF is a computationally efficient method designed to handle large data volumes in an operational setting, and employs an iterative algorithm which makes the method sensitive to spatial variability in data density. Although none of these methods are perfectly suited to our purposes, they do embody a desirable spirit of simplicity and utility.

As we require large interpolated surfaces for a suite of daily meteorological variables, with simulations (typically) of 1-5 years, grids on the order of $500 \times 500$ cells, and incorporating hundreds of observation sites, computational efficiency is an important factor. The faithful application of any of the statistical methods would require at least one parameterization for each variable for each day, and studies suggest that even this would be insufficient for methods such as kriging, given the large and climatically heterogeneous domains of interest. An alternative is to resort to a single parameterization of a statistical method, but this negates what seems to us to be the principal attraction of such methods, that they generate unbiased results, given certain assumptions. Rejecting both of these alternatives, we proceed with the development of a method which lacks both formal optimality criteria and guarantees of unbiasedness, but which can be parameterized once for a given set of observations and applied effectively to the daily observations as often as needed.

In developing our methods, we borrow from the nearest-neighbor method the assertion that the area of relative influence for a given observation should be inversely related to the local observation density; that is, a relatively isolated observation should influence predictions for a larger area than an observation in a data-rich region. The most serious fault of the nearest-neighbor method is that it generates a discontinuous surface, the familiar tesselated surface of Thiessen polygons. We prefer an interpolation surface that is continuous, though we do not impose the condition that it be perfectly smooth, in that its first- and higher-order derivatives are allowed to be discontinuous. From the inverse-distance method we borrow the assertion that relative influence should decrease with increasing distance from an observation. The most serious flaw of the usual implementation of the inverse-distance method is, in our view, that its asymptotic condition forces the surface through all observations, generating spatially anomalous distributions. We desire a method which is, in this sense, a smoother as opposed to an interpolator, in that the resultant surface is not required to pass through the observations.

We adopt as our basic interpolation framework the spatial convolution of a truncated Gaussian filter with a surface containing the horizontal projections of the observation locations. In the spirit of efficiency, truncation of the filter serves to reduce the number of observations included in predictions at a given point: an untruncated filter gives finite weight to all observations at each point of prediction, but the majority of those weights are diminishingly small. Truncation causes a loss of higher-order smoothness, but still results in a continuous surface. We choose a Gaussian function because it is simple to evaluate, and has the desired features of being both an inverse-distance algorithm and a smoothing filter. The descriptions here are given with respect to interpolation over an evenly spaced grid of prediction points, but the same methods could be applied to the generation of predicted values at arbitrarily placed points. 


\section{Methods}

\subsection{Interpolation}

The general form of the truncated Gaussian filter, with respect to its central point, $p$, is

$$
W(r)=\left\{\begin{array}{l}
0 ; \quad r>R_{p} \\
\left.\exp \left[-\left(\frac{r}{R_{p}}\right)^{2} \alpha\right]-\mathrm{e}^{-\alpha} ; \quad r \leq R_{p}\right)
\end{array}\right.
$$

where $W(r)$ is the filter weight associated with a radial distance $r$ from $p, R_{p}$ is the truncation distance from $p$, and $\alpha$ is a unitless shape parameter.

The spatial convolution of this filter with a set of horizontal station locations results, for each point of prediction, in a list of weights associated with observations. Because of the spatially heterogeneous distribution of observations, a constant value for $R_{p}$ results in a large disparity in the number of observations with non-zero weights between points in the least and the most densely populated regions of the prediction grid. We desire a system by which $R_{p}$ can be reduced in data-rich regions, using information from a smaller radius, and increased in data-poor regions. One possibility is the specification of a fixed number of observations to be used at every prediction point, but this can be shown to violate our requirement for a continuous surface. Instead we specify $N$, the average number of observations to be included at each point. $R_{p}$ is then varied as a smooth function of the local station density in such a way that this average is achieved over the spatial domain. The smooth variation of $\boldsymbol{R}_{p}$ ensures a continuous interpolation surface, and is accomplished through the iterative estimation of local station density at each prediction point, as follows:

1. For all grid cells, the same user-specified value, $R$, is used to initialize $R_{p}$.

2. Given $R_{p}$, Eq. (1) is used to calculate weights $W_{i}$, where $i=(1, \ldots, n)$ are observation locations, and the local station density $D_{p}$ (number of stations/area) is then determined as

$$
D_{p}=\frac{\sum_{i=1}^{n} \overline{W_{i}}}{\pi R_{p}^{2}}
$$

where $\bar{W}$ is the average weight over the untruncated region of the kernel, defined as

$$
\bar{W}=\frac{\int_{0}^{R_{p}} W(r) \mathrm{d} r}{\pi R_{p}^{2}}=\left(\frac{1-\mathrm{e}^{-\alpha}}{\alpha}-\right)-\mathrm{e}
$$

3. A new $R_{p}$ is calculated as a function of the desired average number of observations, $N$ : and the most recent calculation of $D_{p}$ as:

$$
R_{p}=\sqrt{\frac{N^{*}}{D_{p} \pi}}
$$


where $N^{*}=2 N$ for the first $I-1$ iterations, and $N^{*}=N$ for the final iteration. This modification of $N^{*}$ is a result of filter truncation, and helps to avoid the occassional occurrence of excessively large station counts in regions of strongly heterogeneous station spacing.

4. The new $R_{p}$ is substituted in Step (2), and Steps (2)-(4) are iterated a specified number of times, $I$. Final values of $R_{p}$ are incorporated in Eq. (1) to generate the interpolation weights $W_{i}$ used in predictions for all days at the point in question.

The interpolation method for a given set of observations and a given prediction grid is defined by the four parameters $R, I, N$, and $\alpha$. Given an arbitrary variable $x_{i}$, measured at each of the $i=(1, \ldots, n)$ observation points, values for the interpolation parameters are specified once and held constant over all days and all prediction points. Taking the case of a single prediction point on a single day, the interpolated value $x_{p}$ is determined in general as

$$
x_{p}=\frac{\sum_{i=1}^{n} W_{i} x_{i}}{\underline{r}}
$$

This general method is refined below, making it specific to predictions of daily temperature extrema and daily total precipitation, incorporating an objective analysis of the influence of elevation differences.

\subsection{Temperature predictions}

Prediction methods for TMAX and TMIN are identical, and we refer here to a general daily temperature variable, $T$. We focus on the generation of a prediction $T_{p}$ at a single point and for a single day, based on observations $T_{i}$, and interpolation weights $W_{i}$, for the $i=(1, \ldots, n)$ measurement sites. Prediction of $T_{p}$ requires a modification of Eq. (2) to include a correction for the effects of elevation differences between the observation points and the prediction point. This correction is based on an empirical analysis of the relationship of $T$ to elevation, which is performed once for each day of prediction.

We introduce a set of transformed variables for the empirical analysis of elevation relationships, under the hypothesis that these relationships may have characteristic spatial and temporal scales which are not well represented by the recorded station elevations $\left(Z_{i}\right)$ and the daily temperature observations $\left(T_{i}\right)$. These new variables are $z_{i}$ and $t_{i}$, a spatial transform of the recorded station elevations and a temporal transform of the daily observations, respectively. In a later section we examine the explicit connection between the transformed and untransformed variables. It should be noted that the use of these transformed variables is limited to the assessment of influence of elevation on predictions of $T_{p}$, and that the untransformed daily observations $T_{i}$ are incorporated in the eventual prediction algorithm.

A weighted least-squares regression is used to assess the relationship between $t$ and $z$. The daily regression is performed over all unique pairs of stations, and the regression weight associated with each point is the product of the interpolation weights associated 
with the stations in a pair. The independent variable is the difference in the transformed elevations associated with a pair of stations, and the dependent variable is the corresponding difference in the transformed temperatures associated with the pair. This gives a regression of the form

$$
\left(t_{1}-t_{2}\right)=\beta_{0}+\beta_{1}\left(z_{1}-z_{2}\right)
$$

where subscripts 1 and 2 indicate the two stations of a unique pair, and $\beta_{0}$ and $\beta_{1}$ are the regression coefficients. We find that this approach is more robust than the simpler method of regressing $t_{i}$ against $z_{i}$, using the $W_{i}$ as regression weights.

$T_{p}$ is then predicted as follows:

$$
T_{p}=\frac{\sum_{i=1}^{n} W_{i}\left[T_{i}+\beta_{0}+\beta_{1}\left(z_{p}-z_{i}\right)\right]}{\sum_{i=1}^{n} W_{i}}
$$

where $z_{p}$ is the elevation assigned to the prediction point, and $T_{p}$ would, in practice, be replaced by either $\operatorname{TMAX}_{p}$ or $\mathrm{TMIN}_{p}$.

\subsection{Precipitation predictions}

Predictions of precipitation are complicated by the need to predict both daily occurrence and, conditional on that result, daily total precipitation. Under the assumption that there is some spatial coherence to the patterns of precipitation occurrence (wet vs. dry) when measured at the time scale of a day, we define a simple binomial predictor of spatial precipitation occurrence as a function of the weighted occurrence at surrounding stations. Taking the case of a single prediction point on a given day, and given observations of daily total precipitation $P_{i}$, and interpolation weights $W_{i}$, we estimate what we loosely refer to as a precipitation occurrence probability, $\mathrm{POP}_{p}$ :

$$
\begin{gathered}
\mathrm{POP}_{p}=\frac{\sum_{i=1}^{n} W_{i} P O_{i}}{\sum_{i=1}^{n} W_{i}} \\
\mathrm{PO}_{i}=\left\{\begin{array}{l}
0 ; P_{i}=0 \\
1 ; P_{i}>0
\end{array}\right\}
\end{gathered}
$$

where the $P O_{i}$ are binomial variables related to observed precipitation occurrence. Daily binomial predictions of precipitation occurrence at a given point, $P O_{p}$, are based on the comparison of $\mathrm{POP}_{p}$ with a specified critical value, $\mathrm{POP}_{\text {crit }}$ :

$$
P O_{p}=\left\{\begin{array}{l}
0 ; \mathrm{POP}_{p}<\mathrm{POP}_{\text {crit }} \\
1 ; \mathrm{POP}_{p} \geq \mathrm{POP}_{\text {crit }}
\end{array}\right\}
$$

POP $_{\text {crit }}$ is held constant for the entire spatial and temporal domain of the simulation.

Conditional on precipitation occurrence $\left(P O_{p}=1\right)$ we proceed with the prediction of 
daily total precipitation, $P_{p}$. Under the same assumptions as outlined for temperature predictions, we introduce the transformed variables $p_{i}$ and $z_{i}$ in the objective analysis of the relationship of precipitation to elevation. Again, we use a weighted least-squares regression, with the same form for the weights and the independent variable as described for temperature predictions. The dependent variable in this case is defined as the normalized difference of the transformed precipitation observations, giving a regression of the form

$$
\left(\frac{p_{1}-p_{2}}{p_{1}+p_{2}}\right)=\beta_{0}+\beta_{1}\left(z_{1}-z_{2}\right)
$$

In generating the predicted daily total, $P_{p}$, we multiply the interpolation weights by the station occurrence variable, $P O_{i}$, giving weight only to those stations which record precipitation for the day in question, as follows:

$$
\begin{gathered}
P_{p}=\frac{\sum_{i=1}^{n} W_{i} P O_{i}\left(\frac{1+f}{1-f}\right)}{\sum_{i=1}^{n} W_{i} P O_{i}} \\
f=\beta_{0}+\beta_{1}\left(z_{p}-z_{i}\right)
\end{gathered}
$$

The form of the precipitation prediction requires that $|f|<1.0$. We introduce another parameter, $f_{\max }(<1.0)$, and force $|f|=f_{\max }$ whenever $|f|>f_{\max }$.

\subsection{Extrapolation smoothing}

We hypothesize that the processes controlling the observed variation of temperature and precipitation with elevation may have characteristic spatial and temporal scales different from those implied by the recorded station elevations or by observations at a daily timestep. For example, it may be that better predictions are obtained from regressions using a spatially smoothed elevation field as opposed to recorded station elevations for the calculation of elevation differences, or that the variation with elevation is better explained using multiple-day running averages of observations as opposed to the daily observations themselves. We introduce the parameters $S_{\mathrm{S}}$ and $S_{\mathrm{T}}$ to describe the spatial and temporal smoothing characteristics for regressions of each variable with elevation.

The parameter $S_{\mathrm{S}}$ describes the degree of spatial smoothing incorporated in the transformation from $Z_{i}$ to $z_{i}$ for a particular variable, and $S_{\mathrm{T}}$ describes the degree of temporal smoothing incorporated in the transformation from $T_{i}$ or $P_{i}$ to $t_{i}$ or $p_{i} . S_{\mathrm{S}}$, measured in kilometers, defines the width of a rectangular region around the presumed location of a particular station for which elevation data from a digital terrain grid are averaged to generate the transformed elevation, $z_{i}$ (see below for a discussion of station location). $S_{\mathrm{T}}$, measured in days, defines the width of a two-sided linearly tapered smoothing filter applied to the time series of $T$ or $P$ to generate $t$ or $p$. Ends of the series are padded with zeros for the purpose of this smoothing filter. In the case of precipitation time series, the smoothing weights for days with no precipitation are set to zero, so the resulting smoothed 
value represents a weighted average of daily precipitation events. This correction is required to avoid the 'constant drizzle' bias associated with a simple smoothing filter.

\subsection{Humidity predictions}

Predictions of humidity are based on the assumption that minimum daily air temperature $\left(T_{\mathrm{m}}\right)$ is a reasonable surrogate for dew-point temperature $\left(T_{\mathrm{d}}\right)$. Tests of this relationship over a wide range of climatic settings indicate that its accuracy decreases with increasing aridity (Kimball et al., 1997), but that in general it is an adequate approximation in the absence of high-quality humidity measurements (Running et al., 1987; Glassy and Running, 1994). We generate estimates of humidity in terms of the average daytime saturation vapor pressure deficit VPD $(\mathrm{Pa})$, as

$$
\mathrm{VPD}=e_{\mathrm{s}}\left(T_{\mathrm{a}}\right)-e_{\mathrm{m}}
$$

where $e_{\mathrm{s}}\left(T_{\mathrm{a}}\right)$ is the saturated vapor pressure (Pa) at the average daytime site temperature $T_{\mathrm{a}}$ $\left({ }^{\circ} \mathrm{C}\right)$, and $e_{\mathrm{m}}$ is the ambient vapor pressure (Pa) as inferred from the assumption that $T_{\mathrm{m}}=$ $T_{\mathrm{d}}$. Vapor pressures are calculated using the Murray (1967) formulation:

$$
\begin{aligned}
& e_{\mathrm{s}}\left(T_{\mathrm{a}}\right)=610.78 \exp \left[\frac{17.269 T_{\mathrm{a}}}{237.3+T_{\mathrm{a}}}\right] \\
& e_{\mathrm{m}}=610.78 \exp \left[\frac{17.269 T_{\mathrm{m}}}{237.3+T_{\mathrm{m}}}\right]
\end{aligned}
$$

Tests of the assumption $T_{\mathrm{d}}=T_{\mathrm{m}}$ have not focused explicitly on the sensitivity of the relationship to variation in $T_{\mathrm{m}}$ owing to local topography. Whereas the mole fraction of water vapor in a well-mixed air column is insensitive to variation in pressure and temperature, partial pressure of water vapor is not. We therefore expect $e_{\mathrm{m}}$ to vary with terrain height, and we assume that the methods described above to analyze the dependence of TMIN on elevation are also applicable to the purpose of estimating variation in $e_{\mathrm{m}}$, and we set $T_{\mathrm{m}}=\mathrm{TMIN}_{\mathrm{p}}$. Similarly, the variation in $T_{\mathrm{a}}$ with respect to elevation has an important effect on VPD, and following Running et al. (1987) we specify

$$
T_{\mathrm{a}}=0.606 \mathrm{TMAX}_{\mathrm{p}}+0.394 \mathrm{TMIN}_{\mathrm{p}}
$$

\subsection{Radiation predictions}

Direct and diffuse components of incident shortwave radiation are calculated on a subdaily timestep (typically $10 \mathrm{~min}$ ) using expressions that analyze the Sun-Earth geometry, including corrections for slope and aspect in complex terrain. Radiative fluxes at the top of the atmosphere are attenuated as a function of atmospheric transmissivity, including corrections for optical air mass and cloudiness. We use the same equations for Sunslope geometry and the empirical treatment of diffuse radiation as described by Hungerford et al. (1989), but substitute the following calculation of daylength, $D L(\mathrm{~s})$, for a flat surface with unimpeded horizons: 
$\mathrm{HSS}=a \cos \left[-\frac{\sin (\mathrm{LAT}) \sin (\mathrm{DECL})}{\cos (\mathrm{LAT}) \cos (\mathrm{DECL})}\right]$

if $\mathrm{HSS}<-\pi$ then $\mathrm{HSS}=-\pi(24 \mathrm{~h}$ daylight $)$

if $\mathrm{HSS}>\pi$ then $\mathrm{HSS}=\pi(0 \mathrm{~h}$ daylight $)$

$D L=2$ HSS $\cdot 13751.0$

where HSS (radians) is the hour angle of sunset, measured from solar noon, LAT (radians) is the latitude, DECL (radians) is the declination angle of the Sun, and the constant 13751.0 converts from radians of hour angle to seconds of daylength. This algorithm reduces errors at high (and southern) latitudes associated with the original daylength algorithm in MTCLIM (Forsythe et al., 1995). DL from this formula is truncated for sloping surfaces as in the original model documentation, but corrections to horizon angles for shading from adjacent terrain elements are not implemented here.

The algorithm of Bristow and Campbell (1984) is used to derive a daily average cloudiness correction to atmospheric transmissivity from the observed diurnal temperature range, DTR, as

$$
\mathrm{PCST}=1.0-\exp \left(-B \mathrm{DTR}^{C}\right)
$$

where PCST is the proportion of clear-sky transmissivity on the day in question, and $B$ and $C$ are empirical parameters (see Glassy and Running (1994) for a discussion of the $B$ and $C$ parameters; here we use the values -0.003 and 2.4 , respectively, by default). This method has been shown to successfully predict a large proportion of the variation in daily radiation fluxes (Running et al., 1987; Glassy and Running, 1994), but no analysis has related its parameters to temperature variability imposed by topographic features. We observe that the environmental lapse rate for minimum temperature is generally of smaller magnitude than that for maximum temperature, and so DTR in a neighborhood will generally decrease with increasing elevation. A strict application of Eq. (5) to the predicted surfaces of DTR $\left(\mathrm{TMAX}_{p}-\mathrm{TMIN}_{p}\right.$ ) would yield consistently lower transmissivity at the higher elevations in a neighborhood of complex terrain. In general, we expect transmissivity to increase with increasing elevation, owing to a reduction in optical air mass, and although some argument could be made for generally more frequent cloudy conditions over high terrain, we think it is unlikely that this phenomenon is responsible for the observed differences in maximum and minimum temperature lapse rates. Our somewhat inelegant solution is to neglect the influence of elevation on $\operatorname{TMAX}_{p}$ and $\mathrm{TMIN}_{p}$ for the purpose of calculating $\mathrm{DTR}_{p}$, performing a simple interpolation of the observed $\mathrm{DTR}_{i}$, using the same form as in Eq. (3) and regarding $\beta_{0}$ and $\beta_{1}$ as 0.0 .

Final predictions of SRAD are made by summing the direct and diffuse shortwave components of radiation over the day and dividing by the daylength, giving the daylight average instantaneous shortwave flux density (in $\mathrm{W} \mathrm{m}^{-2}$ ). 


\subsection{Case-study description and database filtering}

We implemented these methods for a 1 year simulation of daily TMAX, TMIN, PRCP, VPD, and SRAD over an area of $399360 \mathrm{~km}^{2}(832 \mathrm{~km} \times 480 \mathrm{~km})$ in the inland northwestern USA (Fig. 1, topographic detail shown below in Fig. 11(a)). Our region includes southeastern Washington, northeastern Oregon, central Idaho, and southwestern Montana, and was selected to include a diversity of climatic and topographic regions. Elevations over the region range from sea-level to $4000 \mathrm{~m}$. Although there is considerable variation, vegetation is generally grassland, agriculture, and desert at elevations up to $700 \mathrm{~m}$, with coniferous forest dominating at higher elevations, and limited alpine tundra at very high elevations. A notable exception is the dense forest cover ranging from sea-level to about $1000 \mathrm{~m}$ on the west slope of the Cascade Range. Our region extends just to the west of the Cascade Range in Washington and Oregon, encompassing the transition from maritime to continental climates across the Cascade divide. The western Cascade slope is characterized by frequent heavy precipitation, with a gradual increase in annual total precipitation with elevation. The eastern Cascade slope is characterized by a dramatic precipitation gradient, with semi-arid conditions extending $200-300 \mathrm{~km}$ eastward of the foot of the range. Relatively hot and dry conditions prevail across the southern extent of the region, through the northern end of the Basin and Range province, to the Snake River Plain. The eastern portion of the region is dominated by a multitude of Northern Rocky Mountain ranges with elevation ranging from 800 to $4000 \mathrm{~m}$, and very complex topography. Storm tracks are generally from the west, and the west side of this group of ranges receives more precipitation than the east. A minority of storms track from the southeast, and the southern ranges receive the bulk of the moisture from these storms. For the region as a whole, wintertime precipitation comes from large frontal systems, whereas most summer

Location of Study Region: Northwest U.S.

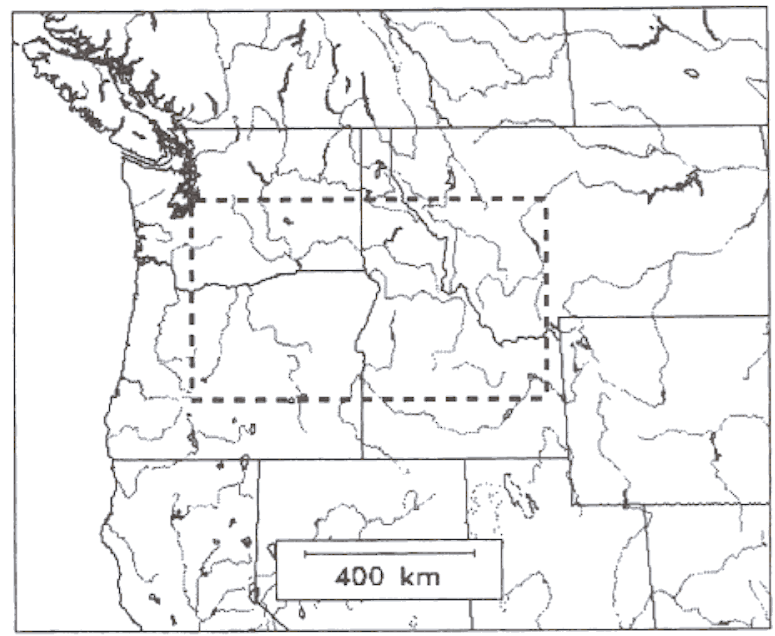

Fig. 1. Northwestern USA, showing state outlines, major river systems, and the approximate boundaries of the study region. 
Table 1

Numbers of stations by type and by inclusion

\begin{tabular}{|c|c|c|c|c|c|c|}
\hline \multirow[t]{2}{*}{ Variable } & \multicolumn{3}{|l|}{ IN } & \multirow{2}{*}{$\frac{\text { USED }}{\text { Total }}$} & \multirow[b]{2}{*}{ WS } & \multirow[b]{2}{*}{ ST } \\
\hline & Total & WS & ST & & & \\
\hline TMAX/TMIN & 280 & 213 & 67 & 436 & 344 & 92 \\
\hline PRCP & 365 & 220 & 145 & 498 & 321 & 177 \\
\hline
\end{tabular}

WS, National Weather Service Cooperative Observers Network station; ST, Natural Resources Conservati Service SNOTEL station.

IN, Inside validation region; USED, inside region or in bordering area.

precipitation is due to local convective activity. An exception is the region west of the Cascade divide, for which frontal precipitation dominates year-round.

Daily meteorological data for 1989 were obtained from the National Climatic Data Center (NWS) and from the Western Regional Climatic Data Center (USDA). Data for stations outside our validation area were incorporated in the predictions for the validation stations, to mitigate the influence of data-sparse edges on interpolation errors. Not all stations recorded all three of the primary variables, and so the number of stations differs somewhat for predictions of temperatures and precipitation. Many SNOTEL stations measured precipitation but not temperature, whereas most NWS stations recorded all three primary variables, resulting in larger numbers of precipitation observations (Table 1).

The original station list was filtered separately for temperature and precipitation data to exclude stations with excessive missing data. Stations were dropped from the initial database if they contained more than 25 days of missing data for the year or if they contained more than five consecutive days of missing data. Stations included in temperature predictions were required to pass these criteria for both TMAX and TMIN, as these are required in tandem for predictions of radiation. Days with missing data for stations passing these criteria were excluded from parameterization and validation analyses.

Station records include the longitude, latitude, and elevation for each station. Longitude and latitude are recorded by the NWS to the nearest arc minute, and elevations are recorded to the nearest meter. An accuracy of \pm 1 arc-min corresponds to a potential error in station location of $3.7 \mathrm{~km}$ for latitude and $2.5 \mathrm{~km}$ for longitude (at $47^{\circ} \mathrm{N}$ ). This is a considerable error, and it creates some difficulties in the registration of station locations to digital terrain maps. For this study we use an equal area projected digital terrain with a resolution of $500 \mathrm{~m}$ (from the US Geological Survey (USGS)) and elevations accurate to about $\pm 6 \mathrm{~m}$. Projection of recorded station longitude and latitude onto our elevation grid results in absolute differences between grid and station elevations which average about $90 \mathrm{~m}$, with a standard deviation of $151 \mathrm{~m}$. Some of this variation is certainly due to the sampling methods used to generate the terrain grid, but we suspect a large proportion is due to inadequate station location data. Given the relative accuracy of station and terrain grid elevations compared with horizontal station locations, we reassigned the station locations to the central point of the $500 \mathrm{~m}$ grid cell in a $4.5 \mathrm{~km} \times 4.5 \mathrm{~km}$ neighborhood around the recorded station location, which minimized the error between recorded and terrain grid elevations. Average absolute difference between grid and station elevation after this location adjustment was $11 \mathrm{~m}$, with a standard deviation of $39 \mathrm{~m}$. The average 
change in horizontal location was $1.9 \mathrm{~km}$, with a standard deviation of $0.7 \mathrm{~km}$. There were no significant differences between precipitation and temperature stations or between NWS and SNOTEL stations in relocation distances.

\subsection{Parameterization and validation}

Cross-validation analysis was used to test the sensitivity of these methods to variation of parameters and to estimate the prediction errors associated with the final selected parameters. The general cross-validation protocol is to withhold one observation at a time from a sample, generating a prediction error for the withheld case by comparison with the observed value, and repeating over all observations in the sample to generate an average prediction error. Our sample in this case is the set of stations which record TMAX, TMIN, or PRCP on a given day. VPD and SRAD are derived from TMAX and TMIN and we are unable to validate them in this framework, having no pertinent observations. We are interested in both the absolute value and the sign of prediction errors generated in this manner. We choose the mean absolute error (MAE) and the bias as our basic prediction error statistics. MAE does not exaggerate the influence of outliers, as does the more common root mean squared error (r.m.s.e.) statistic, and it therefore provides a more robust parameterization framework than r.m.s.e.

The parameterization of these methods is somewhat iterative, in that all parameters relevant to the prediction of one of the primary variables must be specified, to generate cross-validation prediction errors, even though optimal values for some (or, at first, all) of the relevant parameters are unknown. We isolate sets of parameters which are not strongly mutually dependent, and test the covariation of parameters within these sets independently, afterwards combining the results and performing the covariation tests again to correct for the weaker between-set dependences. After values for all parameters are established, a final cross-validation analysis is performed, comparing predictions against observations for both daily values and annual averages (or totals, in the case of precipitation). We give particular attention to daily event frequency histograms for temperature and precipitation, and to the predicted and observed relationships between precipitation occurrence and amount.

\subsection{Spatial scaling analysis}

Our methods are designed to be independent of prediction grid resolution: the process of parameterization and validation is carried out with what are essentially point observations, and we assume that these predictions maintain their validity when applied to the points of a prediction grid. There is another level of abstraction involved in translating these predictions to areal totals or averages as determined by the area of grid boxes centered on the prediction points. We examined the effects of prediction grid resolution on results expressed as areal totals or averages by generating a sequence of increasingly larger prediction grids, ranging in resolution (grid point separation) from $500 \mathrm{~m}$ to $32 \mathrm{~km}$. Digital terrain data with a resolution of $500 \mathrm{~m}$ provided the starting point for this analysis, and we aggregated these data to successively larger grids with resolutions of $1,2,4,8,16$, and 32 $\mathrm{km}$, taking care to maintain the areal correspondence of all grids. Step-wise resampling 
with bilinear interpolation was used to generate grids with progressively larger cell sizes. Daily simulations for 1 year were performed over each grid, and a comparison was made of the areal results.

\subsection{Temporal scaling analysis}

Although these methods are formulated with a daily timestep in mind, they can be transformed to longer (but not shorter) timesteps in a relatively straightforward way. We are encouraged to attempt this transformation because the majority of other published methods operate on monthly or annual timesteps, and because there is continuing interest in the comparison of methods, including ours, with respect to the prediction of annual total precipitation. Here we make a simple analysis comparing the annual average (for temperature) or total (for precipitation) of daily predictions with an implementation that predicts the annual averages or totals from averages or totals of the observations. This transformation is simple for temperature predictions, where we replace the daily observations with the corresponding annual averages, ignoring the $S_{\text {T }}$ parameter. For the case of precipitation, we neglect the occurrence prediction, and proceed with the amount prediction in the same way, assuming all $P O_{i}=1$ and ignoring $S_{\mathrm{T}}$. All other parameters are retained as the optimized values for the case of daily predictions.

\section{Results}

\subsection{Parameterization}

For each of the three primary variables, the interpolation parameters ( $\alpha$, shape parameter; $N$, average number of stations with non-zero weights; $I$, number of station density iteration; $R$, initial truncation radius for iterative density algorithm) are estimated independently. We find that the prediction errors are insensitive to the choice of $R$ for all variables, as long as it is large enough that on the first density iteration at least one station is found inside the truncation radius for each point in the prediction grid. Given the average station density in this case, we assign $R=140 \mathrm{~km}$ for interpolations for all three primary variables. We also find that values for $I>3$ do not generate substantially different smoothed surfaces of $R_{p}$ than $l=3$, and so we use that value by default in all interpolations.

Prediction errors associated with the two remaining interpolation parameters, $\alpha$ and $N$, are found for all three primary variables to exhibit substantial covariation. Prediction errors for TMAX and TMIN were examined for daily predictions and for annual averages of those daily predictions, and in all cases a linear trough of minimized MAE extends from $(N, \alpha)=(25,2.0)$ to $(45,6.0)$ and beyond. Because a low value of $N$ leads to computational savings in the interpolation process, we choose $N=30$ and $\alpha=3.0$ for interpolations of both TMAX and TMIN. The error surfaces for TMAX and TMIN predictions are similar, and an example is given for the daily prediction of TMAX (Fig. 2(a)). Prediction error for annual total precipitation, summed from daily predictions and expressed as a percentage of the total observed precipitation, also shows a linear trough over a range of $N$ and $\alpha$, but 
(a) TMAX: Mean Absolute Error for Daily Predictions $\left({ }^{\circ} \mathrm{C}\right)$

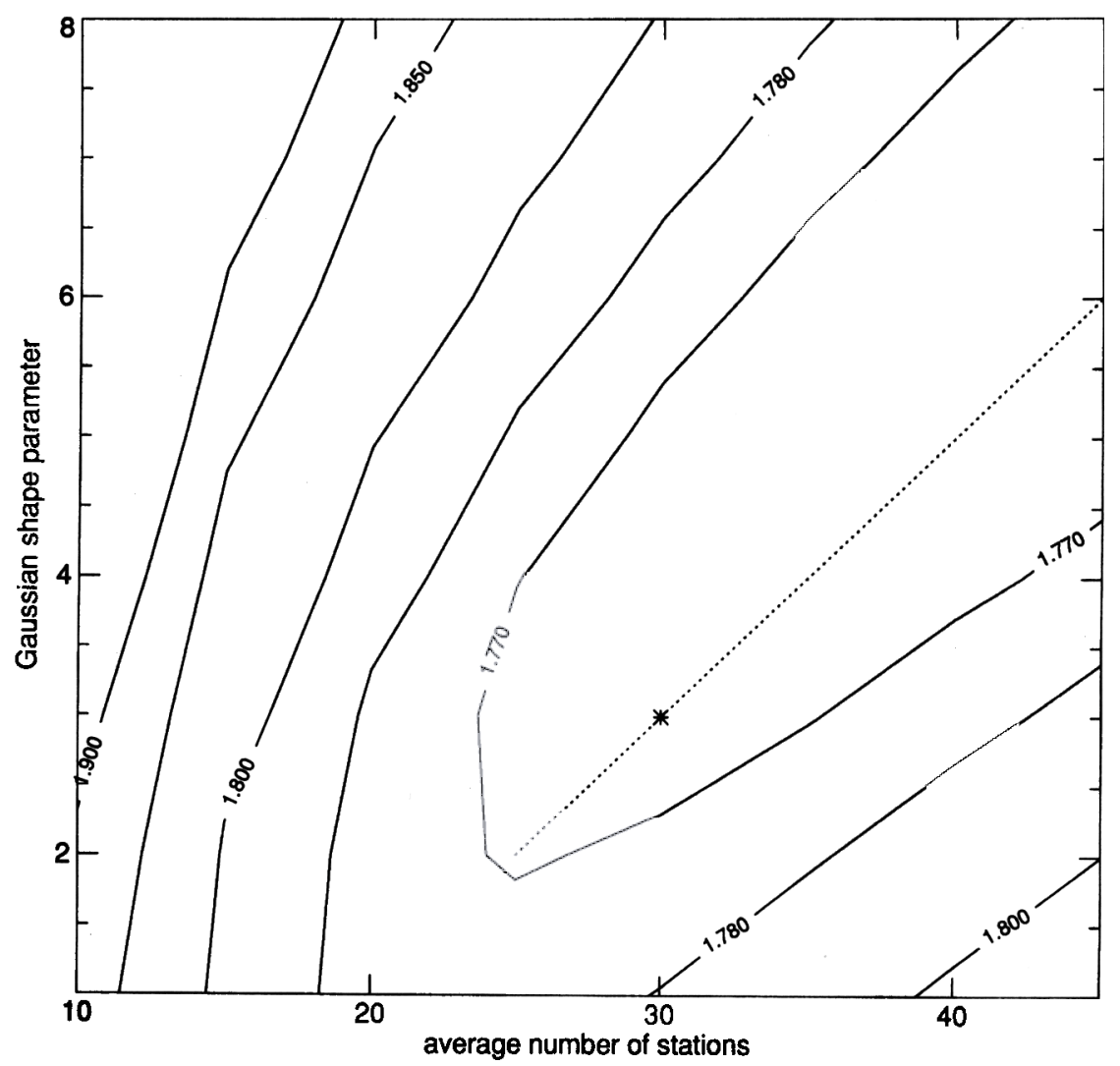

Fig. 2. Contours of mean absolute error plotted against the two most sensitive interpolation parameters, $\alpha$ (Gaussian shape parameter), and $N$ (average number of stations with non-zero weights), for (a) daily predictions of TMAX, and (b) annual totals of daily predictions of PRCP. Dashed lines represent the approximate location of the trough of minimized error, as referenced in the text. *, The coordinate pair selected as the final parameter values.

with optimal values markedly different from those for the temperature predictions (Fig. 2(b)). We choose $N=20$ and $\alpha=6.25$ for the precipitation interpolations.

$S_{\mathrm{S}}$ and $S_{\mathrm{T}}$ were tested in tandem for each of the primary variables. For both TMAX and TMIN, the lowest prediction errors were associated with the use of recorded station elevations in the elevation regressions; prediction error increased linearly with increasing spatial smoothing, $S_{\mathrm{s}}$. Similarly, prediction errors increased linearly for both TMAX and TMIN with increasing $S_{\mathrm{T}}$, with minimum errors obtained using the unsmoothed temperature observations. Prediction errors for precipitation, on the other hand, were found to be substantially reduced by both spatial and temporal smoothing: $S_{\mathrm{S}}$ between 2 and $8 \mathrm{~km}$, and $S_{\mathrm{T}}=5$ days, give optimal results. 
(b)

\section{PRCP: Mean Absolute Error (\% of annual total)}

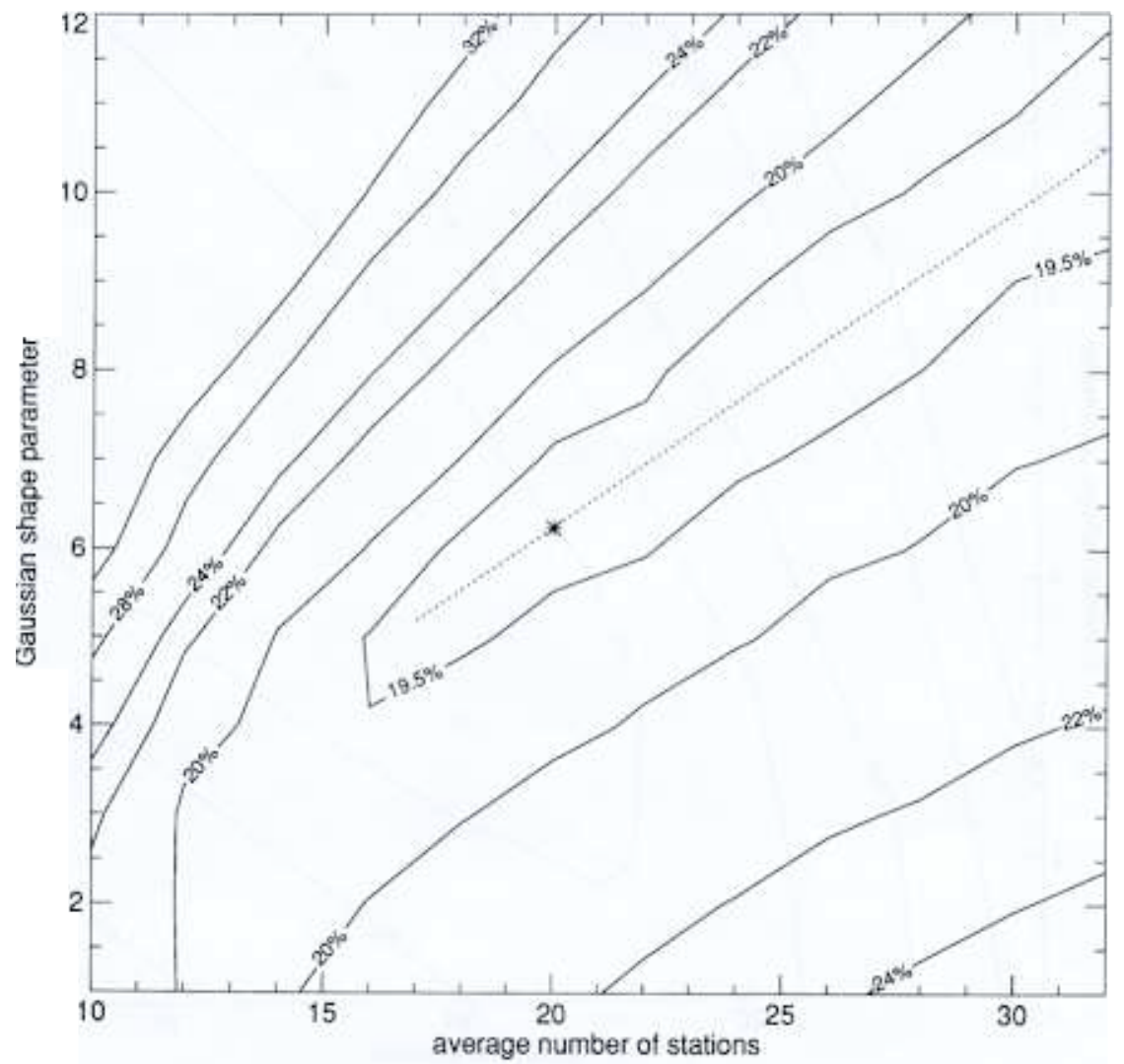

Fig. 2, Continued:

The final two model parameters, $\mathrm{POP}$ crit and $f_{\max }$, are specific to the daily precipitation algorithm. The value for $\mathrm{POP}_{\text {crit }}$ should be close to 0.5 , as it sets the probability of rainfall given the weighted occurrence at a sample of nearby stations: if half or more of these stations record rainfall, we would intuitively predict an event occurrence, otherwise not. Values lower than 0.5 should result in overprediction of events, and therefore a positive bias and a large MAE for rainfall amount. Conversely, values much higher than 0.5 should result in too few predicted events, a negative bias, and again a large MAE for rainfall amount. This is, in fact, what we observe, with the smallest MAE and bias closest to zero at a value of $P O P_{\text {crit }}=0.50$ when the annual total error statistics are measured as centimeters of precipitation. Expressing MAE as a proportion of the observed totals gives an optimal value of 0.55 . As we are unsure which error statistic to favor, we choose a value of 0.52 as a compromise.

As noted in the description of the precipitation algorithm, the parameter $f_{\max }$ is used to constrain the right-hand side of Eq. (4) in the case of large elevation differences and strong 


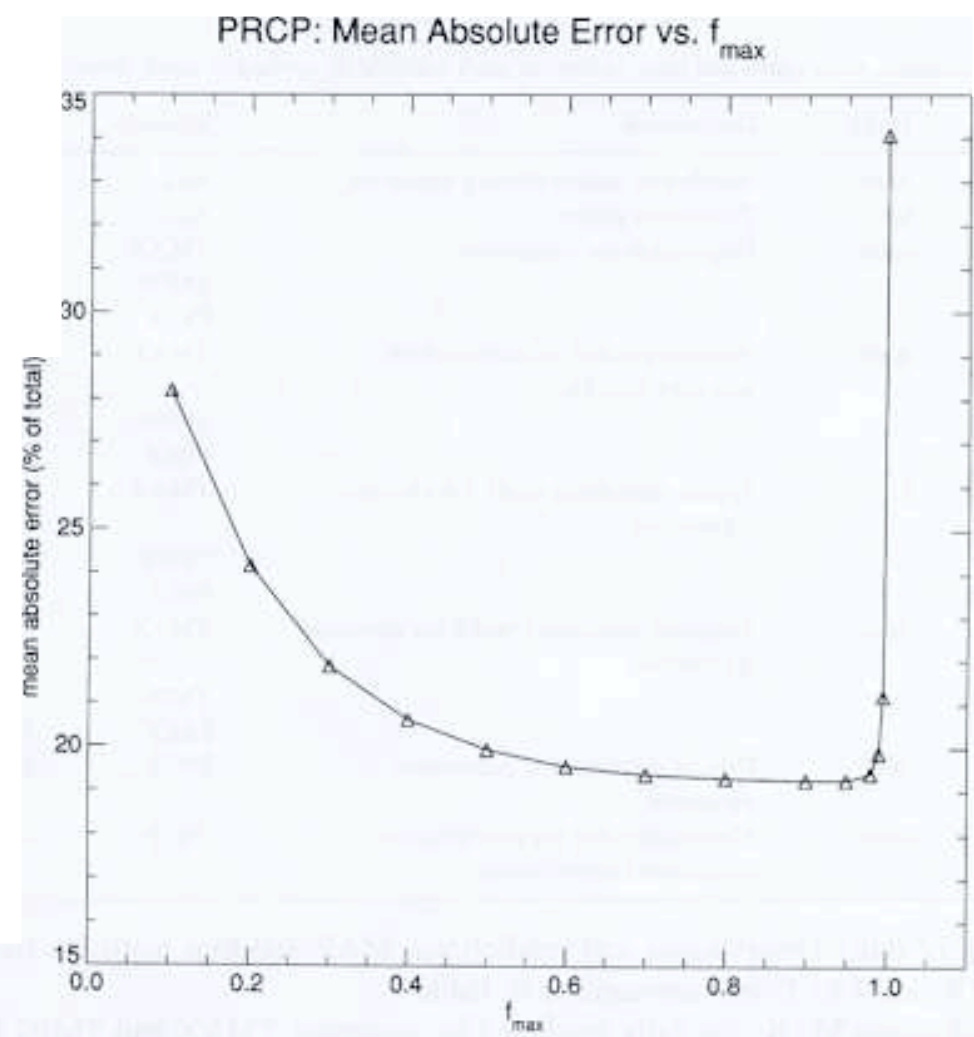

Fig. 3. Influence of $f_{\operatorname{man}}$ on MAE (percentage of annual total) for predicted annual total precipitation. MAE is minimixed at $f_{\max }=0.95$. Similar results are obtained for MAE measured in centimeters.

precipitation-elevation gradients measured at relatively low stations. This parameter essentially embodies our lack of predictive ability for the very highest and wettest terrain. It must assume a value less than 1.0 , and should ideally be set very close to this limit to allow as much information as possible from the regression with elevation to enter the predictions. Values too close to 1.0 will result in excessive predictions at high elevations, increasing the MAE estimated from predicted annual totals. There is a gradual decline in MAE as $f_{\max }$ increases from 0.1 to 0.95 , with a sharp increase in MAE for values approaching 1.0 (Fig. 3). Results are the same for MAE measured in centimeters of total annual precipitation and as proportions of observed totals, and $f_{\max }$ is set to 0.95 for these simulations. All parameters and their values are listed in Table 2.

\subsection{Validation}

Given the parameter values established above (Table 2), we performed a final crossvalidation analysis to assess the accuracy of our methods. We examine here the performance of the daily predictions as well as their annual averages and the frequency 
Table 2

List of all parameters, their units, and their values for each variable as applied in these simulations

\begin{tabular}{|c|c|c|c|c|}
\hline Parameter & Units & Description & Variable & Value \\
\hline$I$ & none & Number of station density iterations & ALL & 3 \\
\hline \multirow[t]{7}{*}{$R$} & km & Truncation radius & ALL & 140 \\
\hline & none & Gaussian shape parameter & TMAX & 3.0 \\
\hline & & & TMIN & 3.0 \\
\hline & & & PRCP & 6.25 \\
\hline & & $\begin{array}{l}\text { Average number of stations with } \\
\text { non-zero weights }\end{array}$ & TMAX & 30 \\
\hline & & & TMIN & 30 \\
\hline & & & PRCP & 20 \\
\hline \multirow[t]{3}{*}{$s_{\mathrm{s}}$} & km & $\begin{array}{l}\text { Spatial smoothing width for elevation } \\
\text { regressions }\end{array}$ & TMAX & 0 \\
\hline & & & TMIN & 0 \\
\hline & & & PRCP & 3.5 \\
\hline \multirow[t]{3}{*}{$S_{\mathrm{T}}$} & days & $\begin{array}{l}\text { Temporal smoothing width for elevation } \\
\text { regressions }\end{array}$ & TMAX & 1 \\
\hline & & & TMIN & 1 \\
\hline & & & PRCP & 5 \\
\hline $\mathrm{POP}_{\text {crit }}$ & none & $\begin{array}{l}\text { Critical precipitation occurrence } \\
\text { parameter }\end{array}$ & PRCP & 0.52 \\
\hline$f_{\max }$ & none & $\begin{array}{l}\text { Maximum value for precipitation } \\
\text { regression extrapolations }\end{array}$ & PRCP & 0.95 \\
\hline
\end{tabular}

distribution of daily observations and predictions. MAE and bias statistics for TMAX, TMIN, DTR, and PRCP are summarized in Table 3.

Cross-validation MAEs for daily predicted vs. observed TMAX and TMIN are $1.8^{\circ} \mathrm{C}$ and $2.0^{\circ} \mathrm{C}$, and MAEs for annual averages of daily estimates are $0.7^{\circ} \mathrm{C}$ and $1.2^{\circ} \mathrm{C}$, respectively. Bias for annual average TMAX and TMIN are $-0.1^{\circ} \mathrm{C}$ and $+0.1^{\circ} \mathrm{C}$, respectively. These errors are very similar in magnitude to those reported for annual predictions from a global database, using a simple interpolation method (Willmott and Robeson, 1995), and to errors for a recent point estimation method (DeGaetano et al., 1995), where a larger

Table 3

Cross-validation error statistics for predictions of daily temperatures, annual average temperatures, and annual total precipitation, averaged over all stations within the validation region, for 1989

\begin{tabular}{|c|c|c|c|c|c|c|}
\hline \multirow[t]{2}{*}{ Variable (units) } & \multicolumn{2}{|c|}{$\begin{array}{l}\text { Daily predictions from } \\
\text { daily observations }\end{array}$} & \multicolumn{2}{|c|}{$\begin{array}{l}\text { Annual predictions from } \\
\text { daily observations }\end{array}$} & \multicolumn{2}{|c|}{$\begin{array}{l}\text { Annual predictions from } \\
\text { annual observations }\end{array}$} \\
\hline & MAE & Bias & MAE & Bias & MAE & Bias \\
\hline $\operatorname{TMAX}\left({ }^{\circ} \mathrm{C}\right)$ & 1.76 & -0.0002 & 0.72 & -0.07 & 0.72 & -0.07 \\
\hline TMIN $\left({ }^{\circ} \mathrm{C}\right)$ & 1.95 & +0.0001 & 1.24 & +0.05 & 1.24 & +0.05 \\
\hline DTR $\left({ }^{\circ} \mathrm{C}\right)$ & 2.30 & -0.0003 & 1.41 & -0.12 & 1.41 & -0.12 \\
\hline $\operatorname{PRCP}(\mathrm{cm})$ & NA & $\mathrm{NA}$ & 13.36 & -2.21 & 12.24 & +0.27 \\
\hline $\operatorname{PRCP}(\%)$ & NA & $\mathrm{NA}$ & 19.27 & +3.03 & 18.47 & +7.09 \\
\hline
\end{tabular}

Precipitation statistics are given both in centimeters of precipitation per year and as percentages of observed annual total.

$\mathrm{NA}=$ not applicable. 
(a) TMAX: Predicted vs. Observed Daily Values

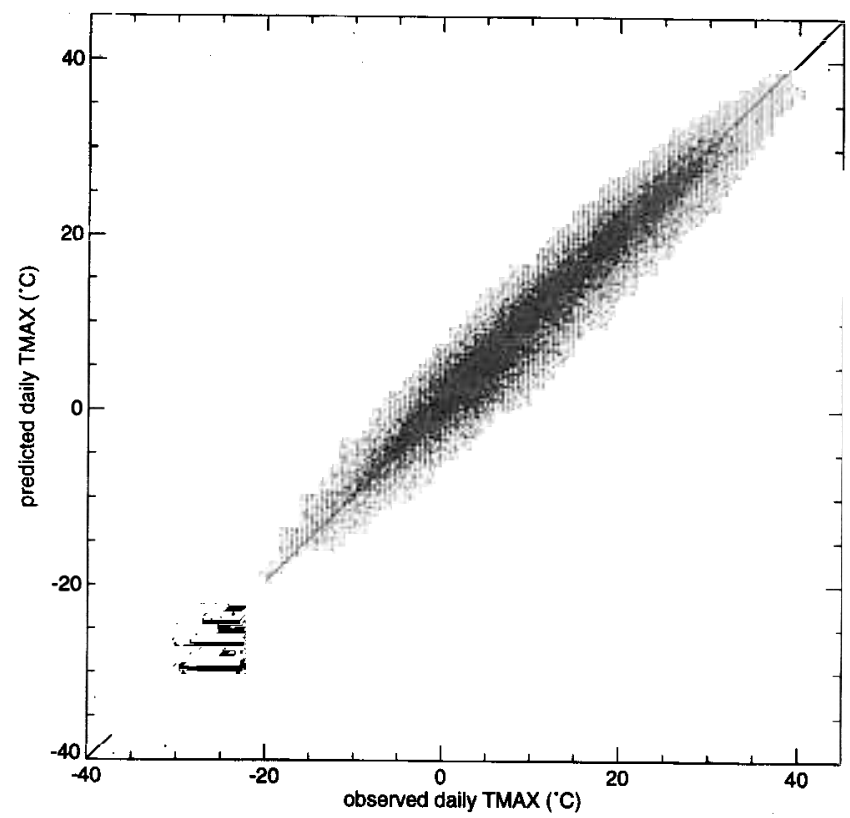

(b) TMIN: Predicted vs. Observed Dally Values
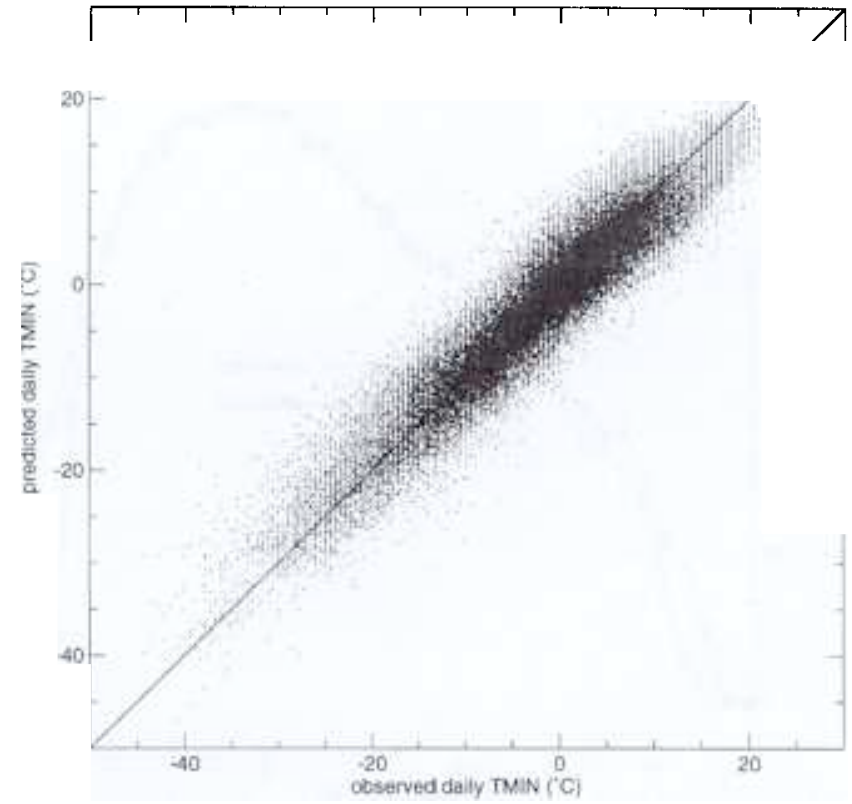
P.E. Thornton et al./Journal of Hydrology 190 (1997) 214-25.

(c) TMAX: Predicted vs. Observed Event Frequency

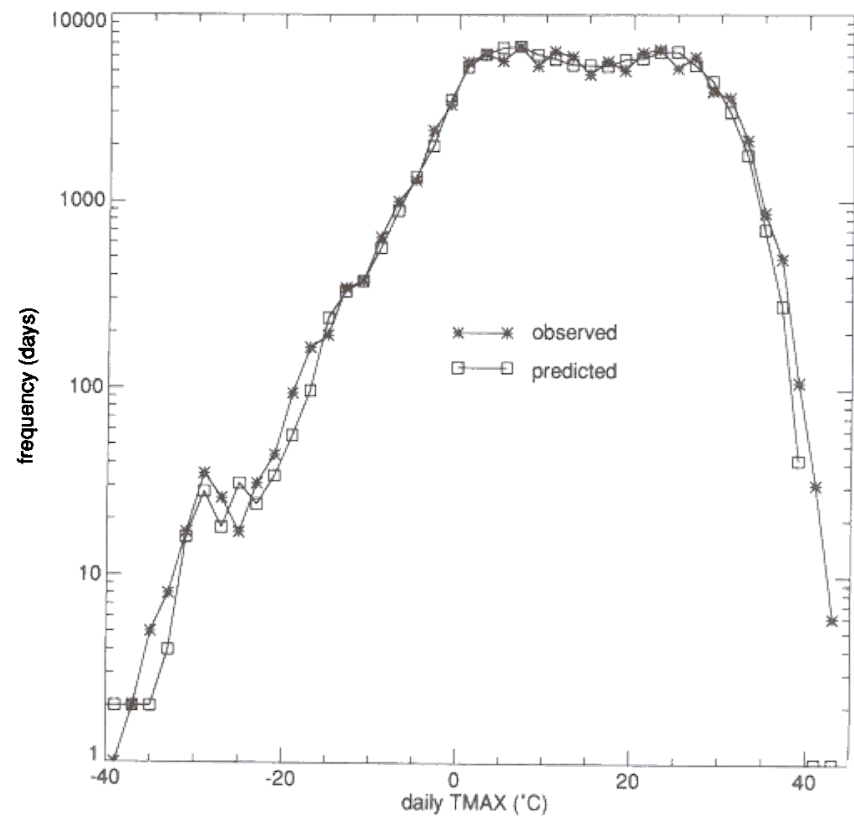

(d) TMIN: Predicted vs. Observed Event Frequency

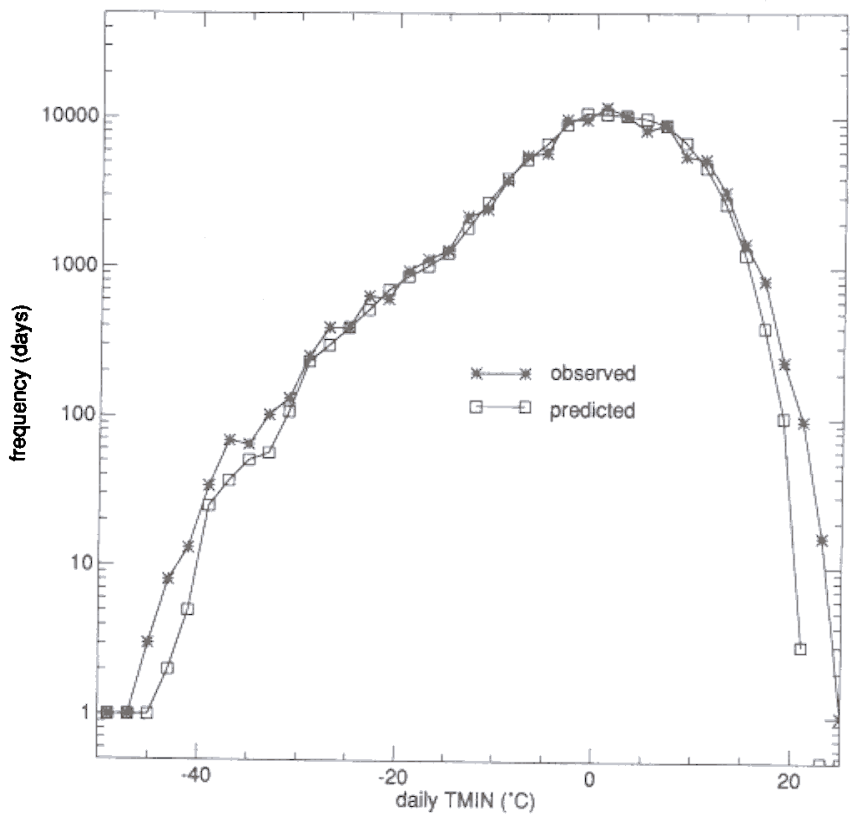


Table 4

Summary of daily precipitation occurrence predictions vs. observations for 365 stations in 1989 (133 225 stati days)

\begin{tabular}{|c|c|c|c|}
\hline \multirow[t]{2}{*}{ Error matrix, $\%$ of station-days } & & Predicted & \multirow[b]{2}{*}{ Wet } \\
\hline & & Dry & \\
\hline Observed & Dry & 65.3 & 10.5 \\
\hline & Wet & 6.2 & 18.0 \\
\hline
\end{tabular}

prediction error for TMIN than for TMAX was also reported. Scatter-plots and frequency histograms of all daily cross-validation temperature predictions show good agreement over most of the observed range, with a tendency to underestimate very high temperatures and to overestimate very low temperatures (Fig. 4).

MAE for daily and annual estimates of DTR are $2.3^{\circ} \mathrm{C}$ and $1.4^{\circ} \mathrm{C}$, respectively, with a bias in the annual averages of $-0.1^{\circ} \mathrm{C}$. The frequency histogram for DTR (not shown) indicates a more serious error in prediction of extreme values than observed for TMAX and TMIN, which is the expected result for an aggregate variable. This information is included to give a general notion of the likely errors in the radiation routine as a result of errors in DTR: as corrections to DTR for elevation are neglected in the radiation algorithm there are no strictly applicable error statistics.

The validation of the daily precipitation model is somewhat more involved, owing to the influence of daily occurrence predictions. Strict estimates of daily error, as performed for temperature variables, are unenlightening: it is a curious fact that these errors are minimized by assuming no precipitation whatsoever, because, for the climate in question, this is true on a large majority of station-days. MAE estimated from annual totals of daily predictions, as used in the model parameterization, is a much more robust indicator of model performance, but it sheds no light on the relationship between occurrence and amount. For example, a reasonable annual total could be attained with a large number of very small daily events or with a small number of large events, and both cases are probably inaccurate. Assessments of both annual totals and the relationship between occurrence frequency and amount are required.

MAEs obtained from simple differences in annual totals and from percentages of observed annual totals are $13.4 \mathrm{~cm}$ and $19.3 \%$, respectively. Prediction errors increase with increasing observed totals, but are normally distributed on a log scale (Fig. 5(a)). Estimated biases in annual totals are $-2.1 \mathrm{~cm}$ and $+3.0 \%$, by the two methods, and the difference in sign between these two estimates reflects the compromise in the parameter-

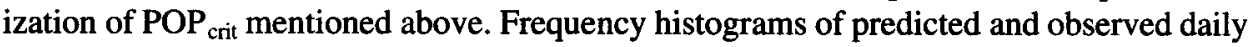
precipitation amounts (Fig. 5(b)) show a small but consistent underprediction of event frequency in the middle of the range $\left(2-8 \mathrm{~cm} \mathrm{day}^{-1}\right)$, and an overprediction of event

Fig. 4. Scatter-plots and frequency histograms for daily cross-validation predictions and observations of temperature extrema: (a) TMAX scatterplot, (b) TMIN scatterplot, (c) TMAX frequency histograms, (d) TMIN frequency histograms. Continuous line in scatterplots shows 1:1 relationship. Vertical histogram axes are log. scaled to show detail in the ends of the distributions. 
(a) PRCP: Predicted vs. Observed Annual Total

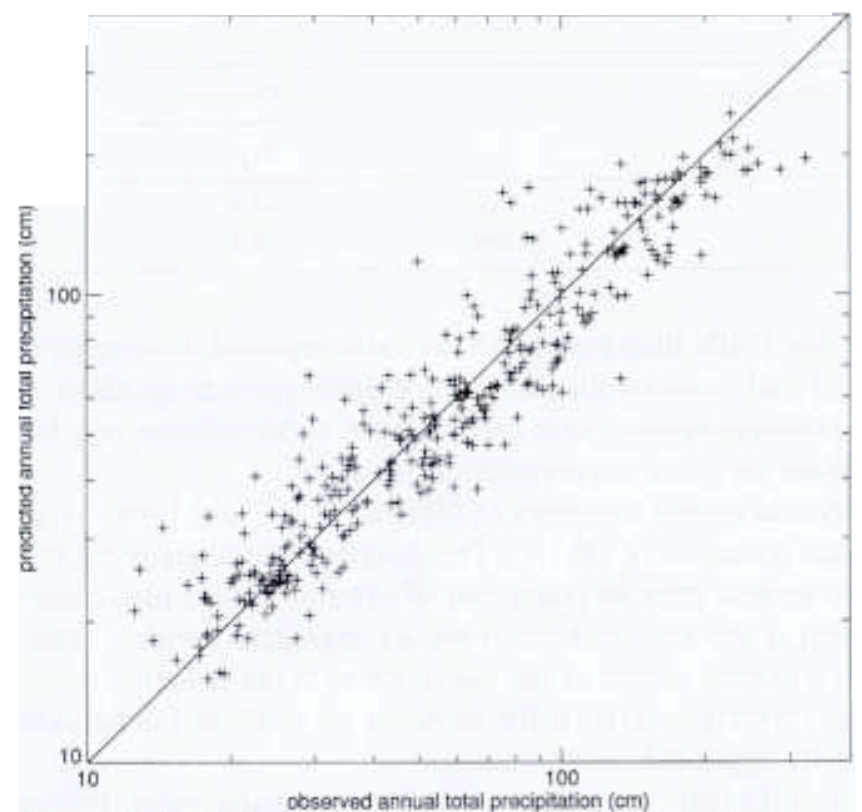

(b) PRCP: Predicted vs. Observed Event Frequency

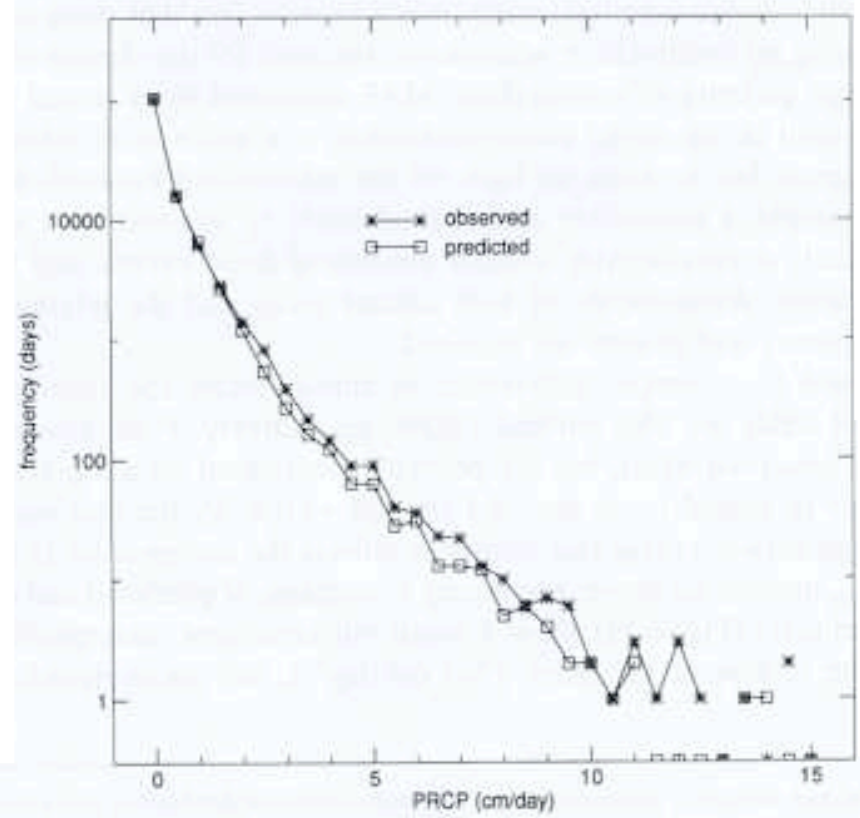

Fig. 5, (a) Scatterplot of annual total of daily cross-validation predictions and observations of precipitation (loglog scales); continuous line shows 1:1 relationship. (b) Frequency histogram of daily cross-validation predictions and observations of precipitation; vertical axis is log-scaled to show detail for extreme events. 
Spatial Frequency of Dry Stations: Predicted vs. Observed

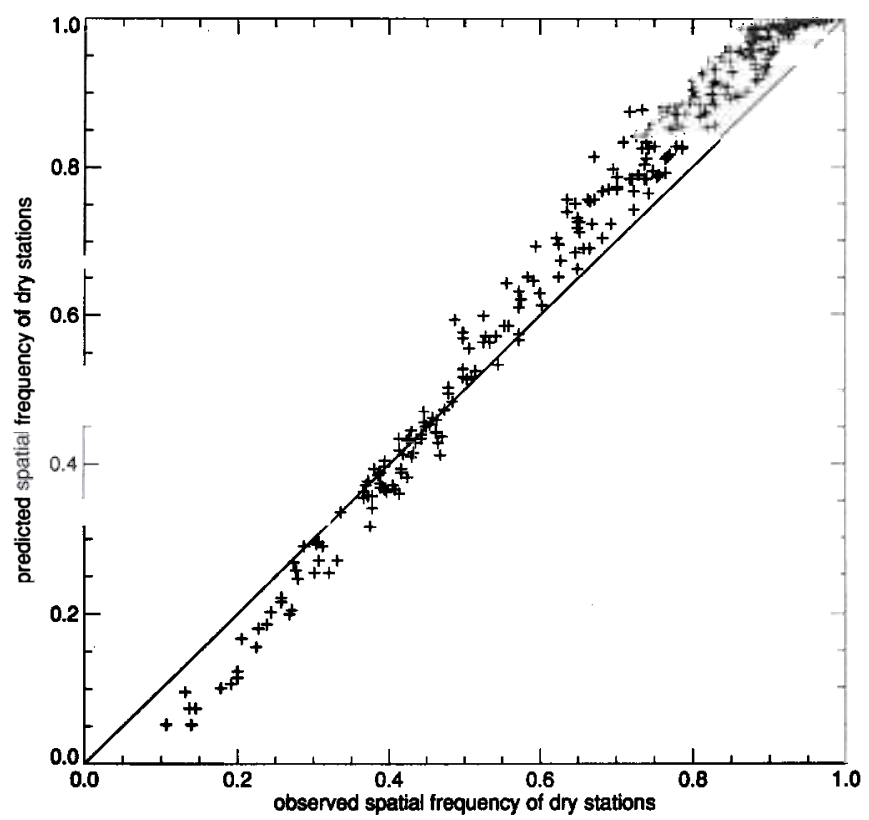

Fig. 6. Predicted vs. observed spatial frequency of dry stations (scaled as a proportion of all stations); one poin per day.

frequency around $1 \mathrm{~cm} \mathrm{day}^{-1}$. Our methods accurately reproduce the frequency of both dry days and extreme precipitation events.

Daily precipitation occurrence predictions are summarized in Table 4. Of all simulated station-days, we predicted $71.5 \%$ dry and $28.5 \%$ wet, compared with observed values of $75.8 \%$ dry and $24.2 \%$ wet, with an overall success rate for occurrence prediction of $83.3 \%$ ( $91.4 \%$ for dry days, and $63.0 \%$ for wet days).

As an estimate of our ability to reproduce, for a given day, the relative proportions of wet and dry areas in a large region, we plot the predicted vs. observed spatial frequency of dry stations (Fig. 6). We underestimate the frequency of dry stations on days with widespread precipitation, and we overestimate the frequency of dry stations on days with scattered precipitation. We predict the spatial frequency correctly in the middle of the range, when about half of the stations are wet and half dry. Fig. 6 illustrates the accuracy of the average predicted occurrence distribution over the study area on any day, but does not provide any information on the accuracy of the predicted spatial distribution of occurrence. Plotting the spatial frequency of correct occurrence predictions against the observed spatial frequency of dry stations (Fig. 7) shows that our predictions have the best spatial accuracy on very wet and very dry days, and suffer in the middle range where wet and dry stations are mixed evenly.

As an indication of the accuracy with which our methods reproduce the observed relationship between daily areal coverage of precipitation and daily average precipitation 
Successful PO Predictions vs. Frequency of Dry Stations

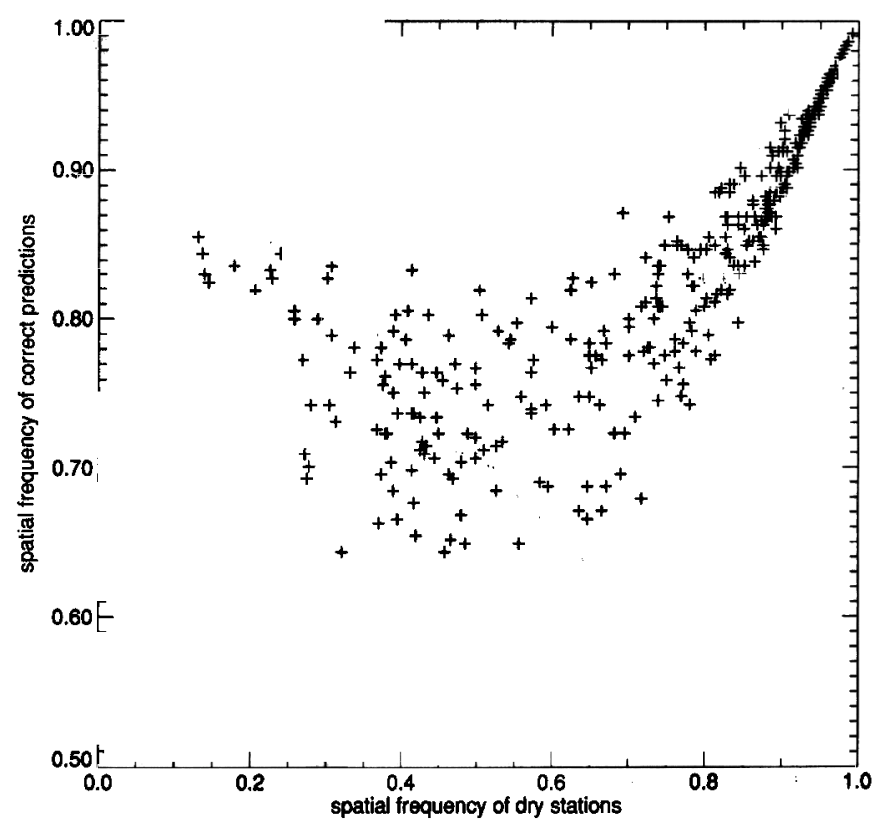

Fig. 7. Spatial frequency of correct precipitation occurrence predictions plotted against the spatial frequency of dry stations (frequencies scaled as proportions of all stations); one point per day.

intensity, we plot observations and predictions of the daily total precipitation (averaged over stations) vs. the daily proportion of wet stations. The predicted distribution matches the observations very well, with the exception of the underprediction of the proportion of wet stations on very dry days (Fig. 8).

\subsection{Spatial and temporal scaling analysis}

We find that areal means (for temperature predictions) or totals (for precipitation) over the study region are preserved for a wide range of prediction grid resolutions. Areal mean annual average TMAX and TMIN of $12.9^{\circ} \mathrm{C}$ and $-6.7^{\circ} \mathrm{C}$, respectively, and areal mean annual total PRCP of $65.2 \mathrm{~cm}$ are obtained for independent simulations over nested prediction grids ranging in grid-point spacing from $500 \mathrm{~m}$ to $32 \mathrm{~km}$. A shift to larger grid spacing reduces the range of variation over the grid, as a result of reduced variability in the elevations represented by the grid points.

Temporal scaling of the temperature outputs is perfect for all timesteps larger than 1 day: the same result is obtained by generating daily predictions from daily observations and averaging over a longer time period as is obtained by generating predictions for the longer time period directly from averaged observations for the period. This result is a consequence of the linear nature of the prediction algorithms. Temporal scaling of the precipitation algorithms is confounded somewhat by the binary predictions of precipitation 


\section{Daily Average PRCP vs. Proportion of Wet Stations (log:log)}

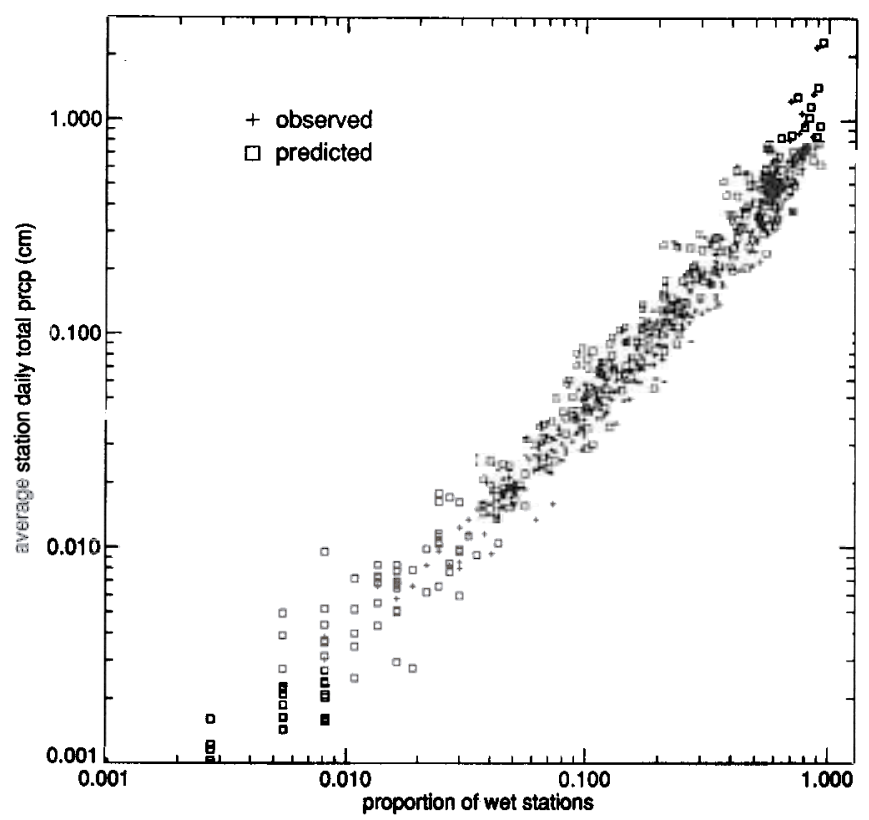

Fig. 8. Precipitation intensity vs. areal coverage for spatial averages of predictions and observations on each day ( $\log -\log$ scales).

occurrence at the daily timestep. For predictions of annual totals from observed annual totals, we obtain cross-validation MAEs of $12.2 \mathrm{~cm}$ and $18.5 \%$, lower by $1.2 \mathrm{~cm}$ and $0.8 \%$ than MAEs obtained from totals of daily predictions. These differences between the two time scales of prediction are significant at the 0.01 level for both methods of estimating MAE, and provide a crude estimate of the contribution of occurrence prediction error to the daily prediction errors. There is no trend in the differences between the daily and annual predictions over the range of predicted annual totals (Fig. 9), an indication that the prediction occurrence algorithm is not introducing significant biases which are related to precipitation intensity. Biases for the annual predictions are $+0.3 \mathrm{~cm}$ and $+7.1 \%$.

\subsection{Example output}

It is not possible here to illustrate the daily sequences of predicted surfaces, but we are able to provide spatial output summaries. We have selected a prediction grid point spacing of $2 \mathrm{~km}$ for these example results. As an example of the temporal variability in the diagnosed relationships of TMAX, TMIN, and PRCP to elevation, time series of the spatially averaged regression slopes $\left(\beta_{1}\right)$ are shown in Fig. 10. Annual aggregates of the predicted daily surfaces are shown in Fig. 11, with TAVG, VPD and SRAD shown as annual averages, $P O$ shown as the number of wet days for the year, and PRCP shown as the annual total. 
Annual vs. Daily Predictions of Annual Total PRCP

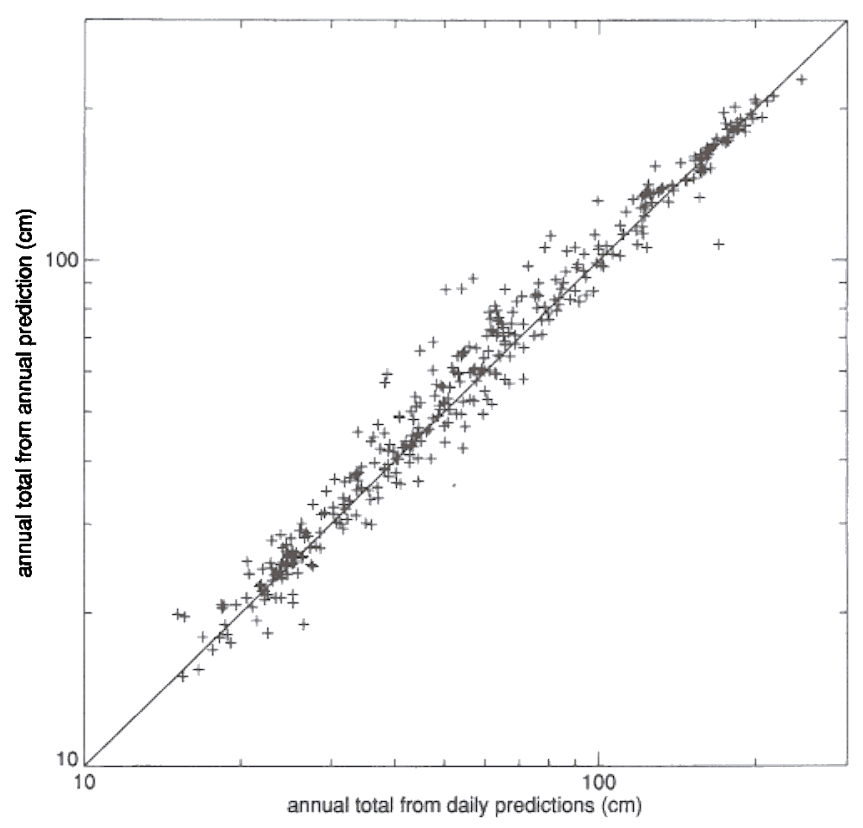

Fig. 9. Influence of temporal scaling on predictions of annual total precipitation: annual totals from daily predictions plotted against annual totals from a single annual prediction (log-log scales).

\section{Discussion}

The methods described and implemented here are essentially ad hoc, in that their design has been guided by the particular needs of regional hydroecological process simulation. However, as one of our needs is that the methods be applicable to multiple variables and purposes (e.g. the use of the same interpolation weights to drive predictions of both precipitation occurrence and intensity), we arrive at the curious case of an ad hoc approach which is also general. Much of this generality stems from a conscious decision to allow the observations to dictate, to a large extent, the interpolation and extrapolation parameters. For example, whereas Daly et al. (1994) have stressed the importance of an explicit accounting of the influence of leeward and windward aspect in distributing precipitation in mountainous terrain, we find that our methods faithfully reproduce the extreme differences in precipitation gradient on the west and east sides of the Cascade Range without recourse to anisotropic filtering criteria (Fig. 11(e)). This is not to suggest that prevailing wind conditions are an unimportant component of precipitation distribution, but rather to call attention to the ability of simple, isotropic methods to extract such information from a topographic neighborhood. The same empirical arguments for simplicity can also be made with respect to the relationship between precipitation occurrence (or areal coverage) and intensity. In a discussion of stochastic precipitation predictions, Hutchinson (1995) noted the disparity in spatial and temporal scales of correlation between event-based 
(a) Temperature vs. Elevation Regression Slopes

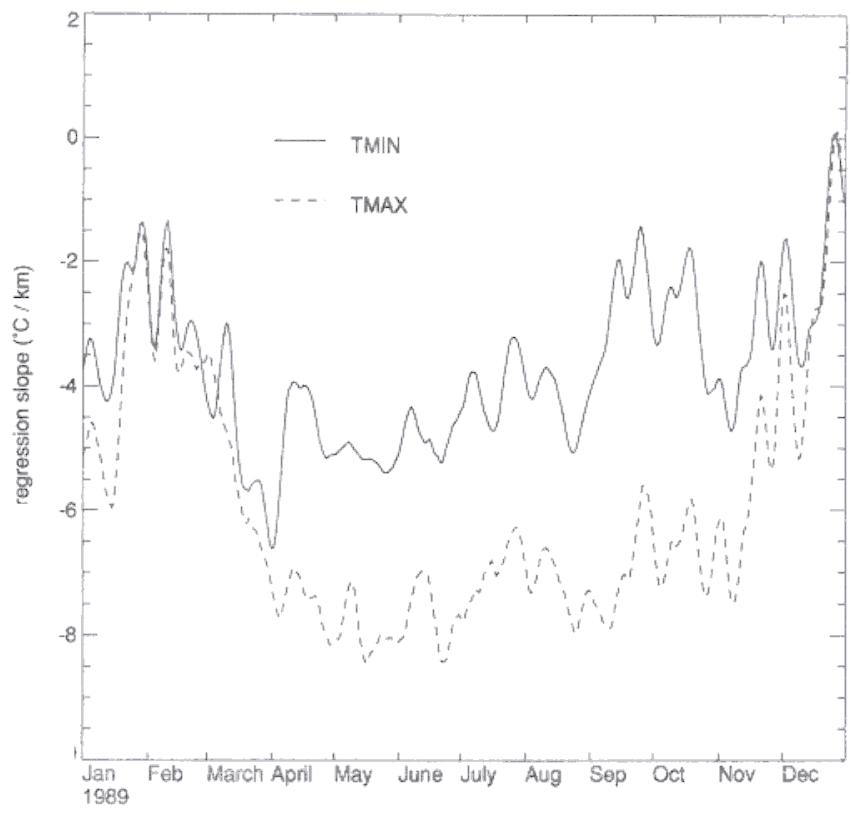

(b) Precipitation vs. Elevation Regression Slope

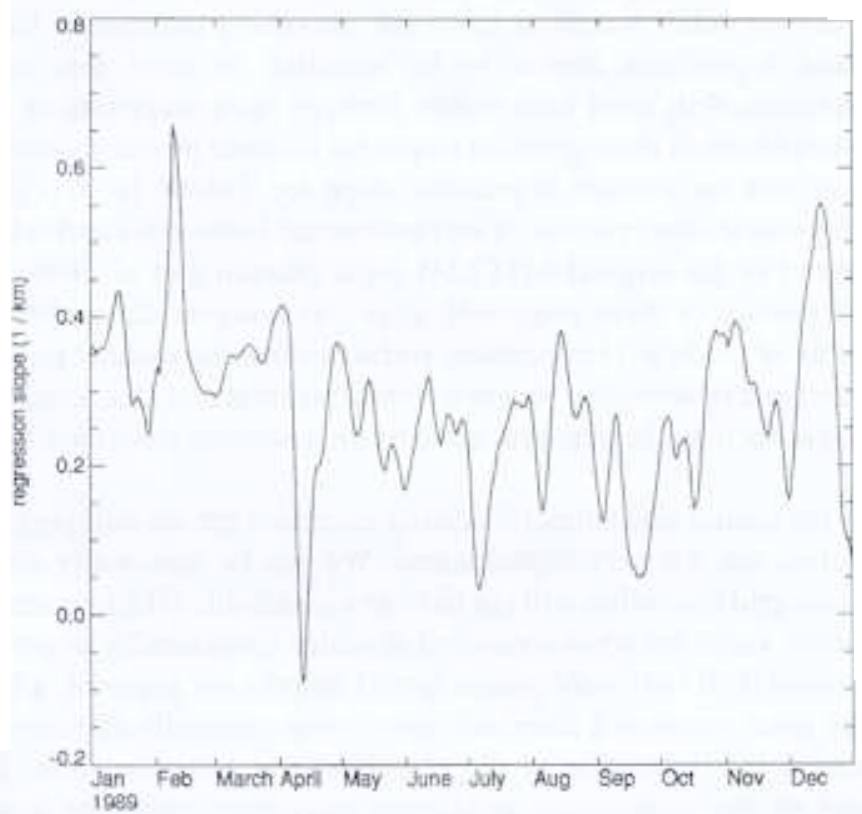

Fig. 10. Time series of spatially averaged regression slopes $(\beta$,$) for (a) TMAX and TMIN, and (b) PRCP. Only$ grid points for which the weighted average difference in elevation between observation and grid-zell elevation exceeds $200 \mathrm{~m}$ are included in this average. This restricts the spotial average to areas of eomplex tertain. For clarity, an 11 day tupered smoothing filter has been applied to these time series. 
precipitation occurrence and intensity, and suggested that these processes should be treated separately in spatial interpolations. We find, however, that an extremely simple abstraction of occurrence likelihood and intensity, derived from the same spatial and temporal interpolation parameters, gives reasonable results for a daily timestep (Fig. 5(b), Fig. 6 and Fig. 8). These results are due in part to the use of observed as opposed to modeled or stochastic sequences of daily precipitation: even at densities which would generally be considered far too low to resolve important spatial precipitation features, enough information is retained to produce realistic daily time series of both occurrence and intensity from a single, point-wise isotropic interpolation.

The point-wise isotropic nature of our method is in contrast to its spatially varying scaling properties. The sensitivity of our method to local observation density, through the incorporation of the iterative station density algorithm, is a trait it shares with the smoothing splines method as implemented by Hutchinson (1995), the recursive filter objective analysis of Hayden and Purser (1995), and to some extent the topographic facet logic of Daly et al. (1994). We suggest that it is this feature which most distinguishes these methods from other, both sophisticated and simple, methods.

The results of our tests of the characteristic spatial and temporal smoothing scales associated with regressions against elevation are in agreement with results reported for precipitation-elevation regressions by Daly et al. (1994), who suggested an optimal DEM cell-size of 4-10 km, compared with our result of 2-8 km for the smoothing width. We have found no relevant studies with which to compare our results for temporal smoothing width, but we were somewhat surprised at the result that smoothing windows longer than 5 days did not noticeably improve precipitation predictions. Similarly, we found no relevant references for either spatial or temporal smoothing parameters for temperature regressions. These regressions appear to be sensitive to local topography, and to atmospheric conditions with short time scales. Perhaps most surprising of all is the systematic temporal variation in the regression slopes for all three primary variables (Fig. 10). It is worth noting that the average regression slope for TMAX is $-6.0^{\circ} \mathrm{C} \mathrm{km}^{-1}$, which agrees well with general observations of environmental lapse rates and with the default lapse rate employed in the original MTCLIM logic (Running et al., 1987). We plan to examine the relationship of these diagnosed slopes to synoptic atmospheric conditions; temporal sequences of predicted temperature surfaces show the distinct passage of fronts, and we hope to derive a relationship between frontal position and characteristic regression slopes using a logic such as the synoptic classification scheme described by Pielke et al. (1987).

The results of the spatial and temporal scaling exercises are encouraging, although the exercises themselves are not very sophisticated. We can be reasonably certain that our choice of prediction grid resolution will not have any noticeable effect on areal averages or totals of the primary variables when measured at scales considerably larger than the grid resolution. For example, if only very coarse spatial outputs are required, a widely spaced grid will give the same result as a finer and more computationally expensive grid, given that care is taken in the translation of the elevation data between grids. The temporal scaling properties of the temperature prediction algorithms eliminate any uncertainty associated with predictions at different timesteps. The close agreement between predicted annual total precipitation at daily and annual timesteps (Fig. 9) gives us some confidence 


\section{Study Region: Elevation and Station Locations}

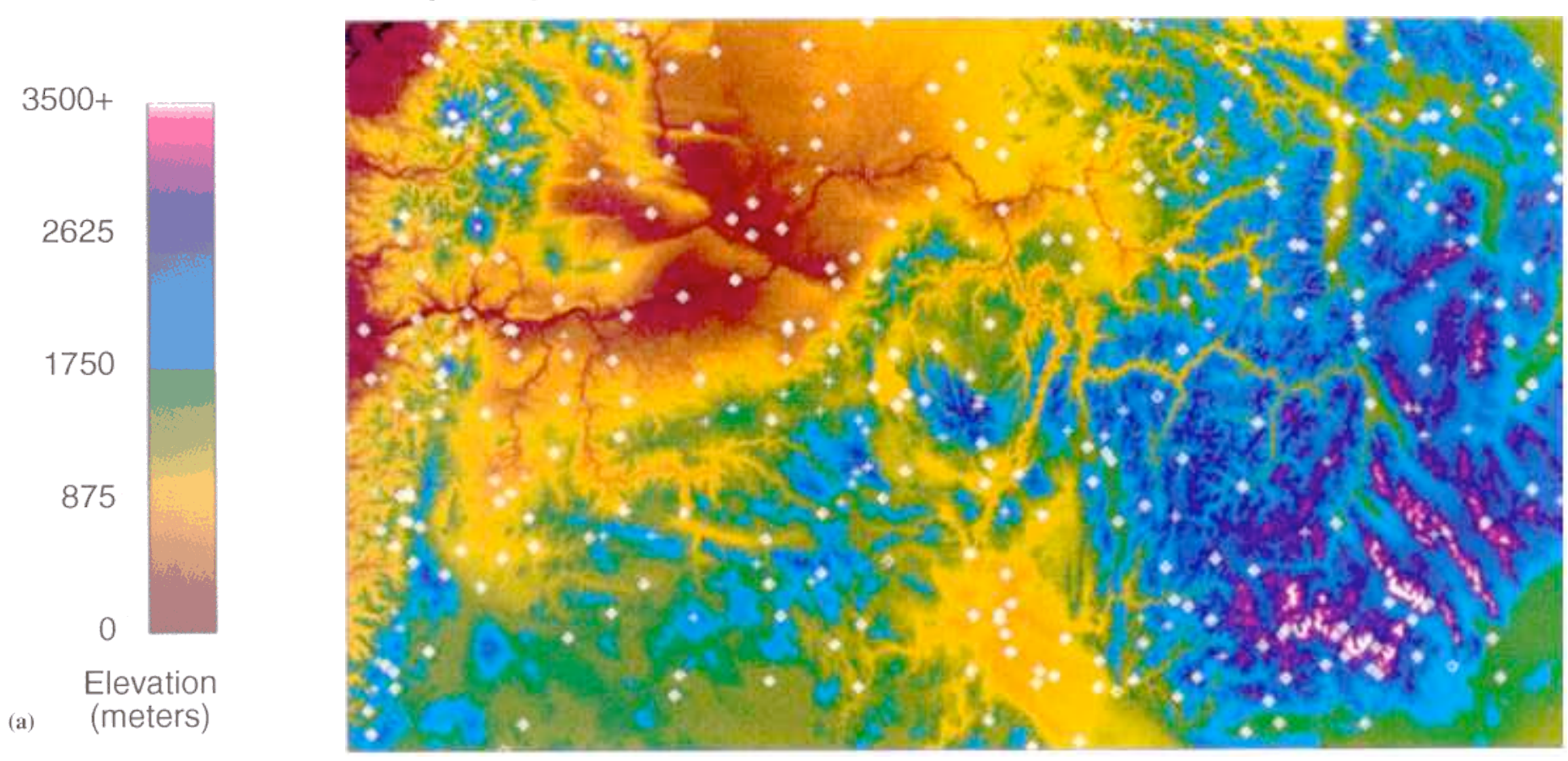

Fig. 11. (a) Detail of study region, showing topography and station locations. Crosses indicate PRCP stations, boxes indicate TMAX and TMIN stations, and overlapping symbols indicate the observation of all variables at the site. Major topographic features are the Cascade Range on the far western edge of the region, Blue Mountains center, Salmon River Mountains, Bitterroot range, and other associated ranges of the Northern Rocky Mountains to the east, and the Snake River Plain south-east to south-central. Remaining color plates show annual averages or totals of selected outputs: (b) annual average daytime temperature; (c) annual average daytime VPD; (d) annual total of wet days; (e) annual total precipitation; (f) annual average of the daylight average incident shortwave radiative flux density. Scale bars shown for (c) and (f) apply to all figures. The same general color scheme has been employed to help discern the differing influence of topographic and geographic effects on the various annual average outputs. 

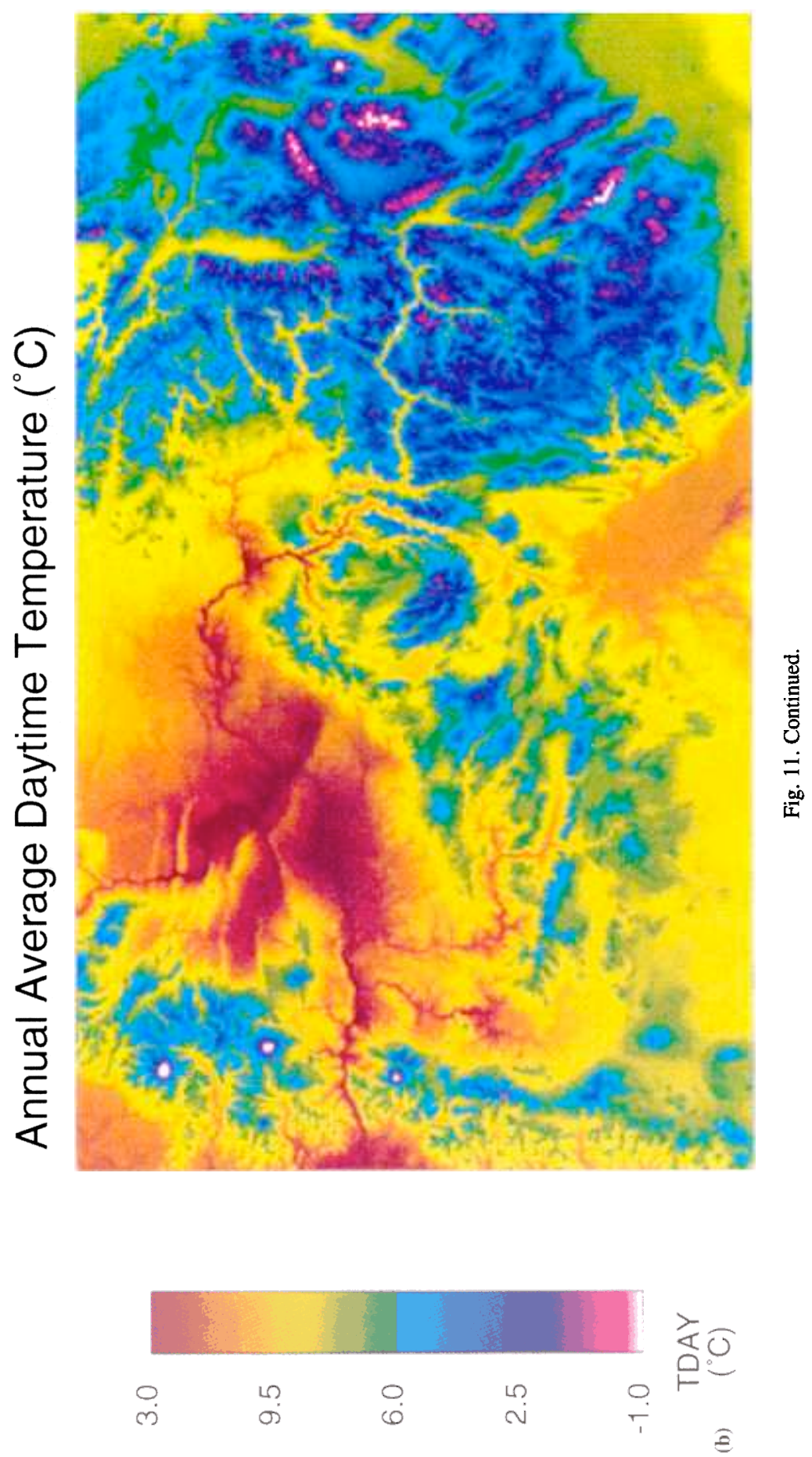


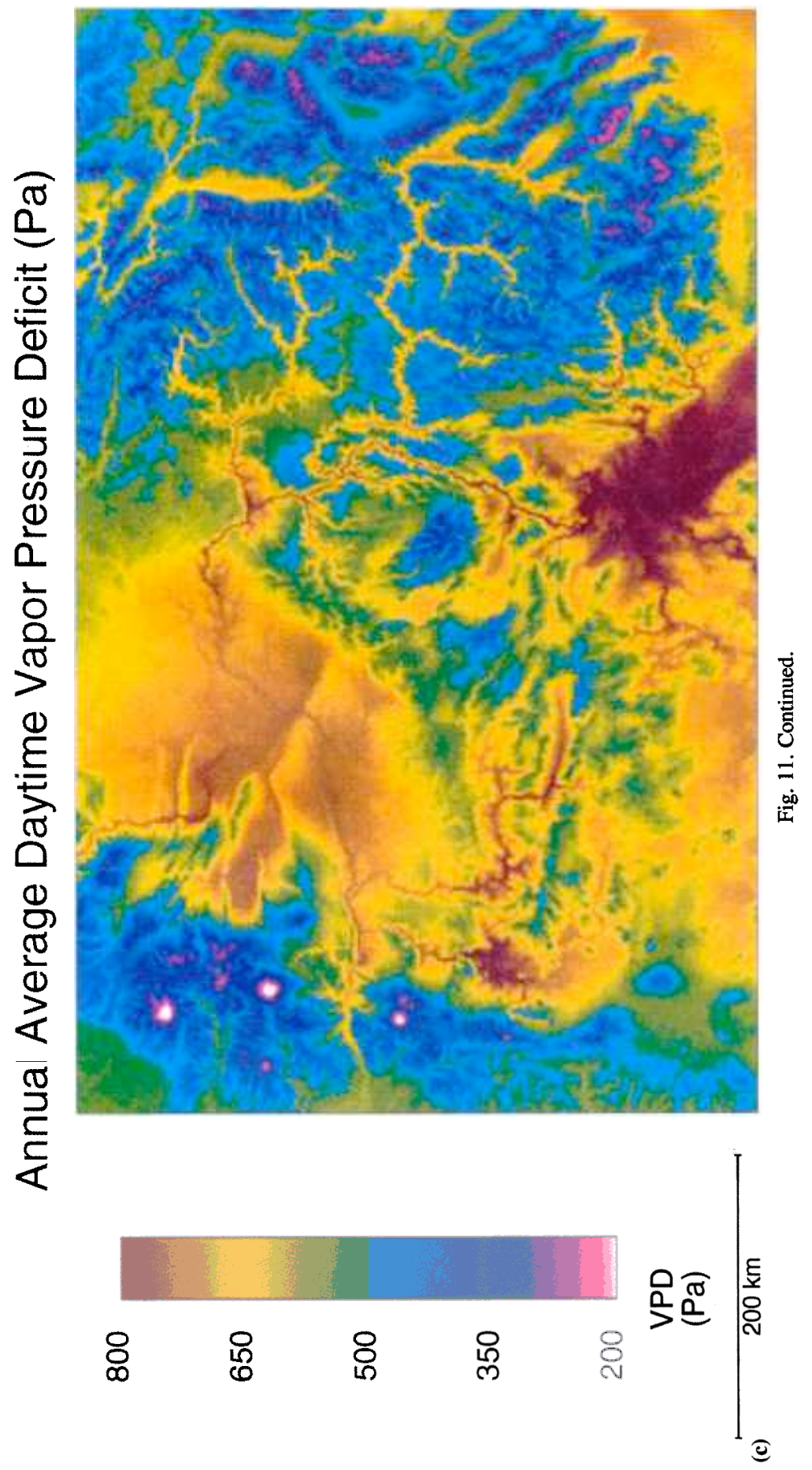



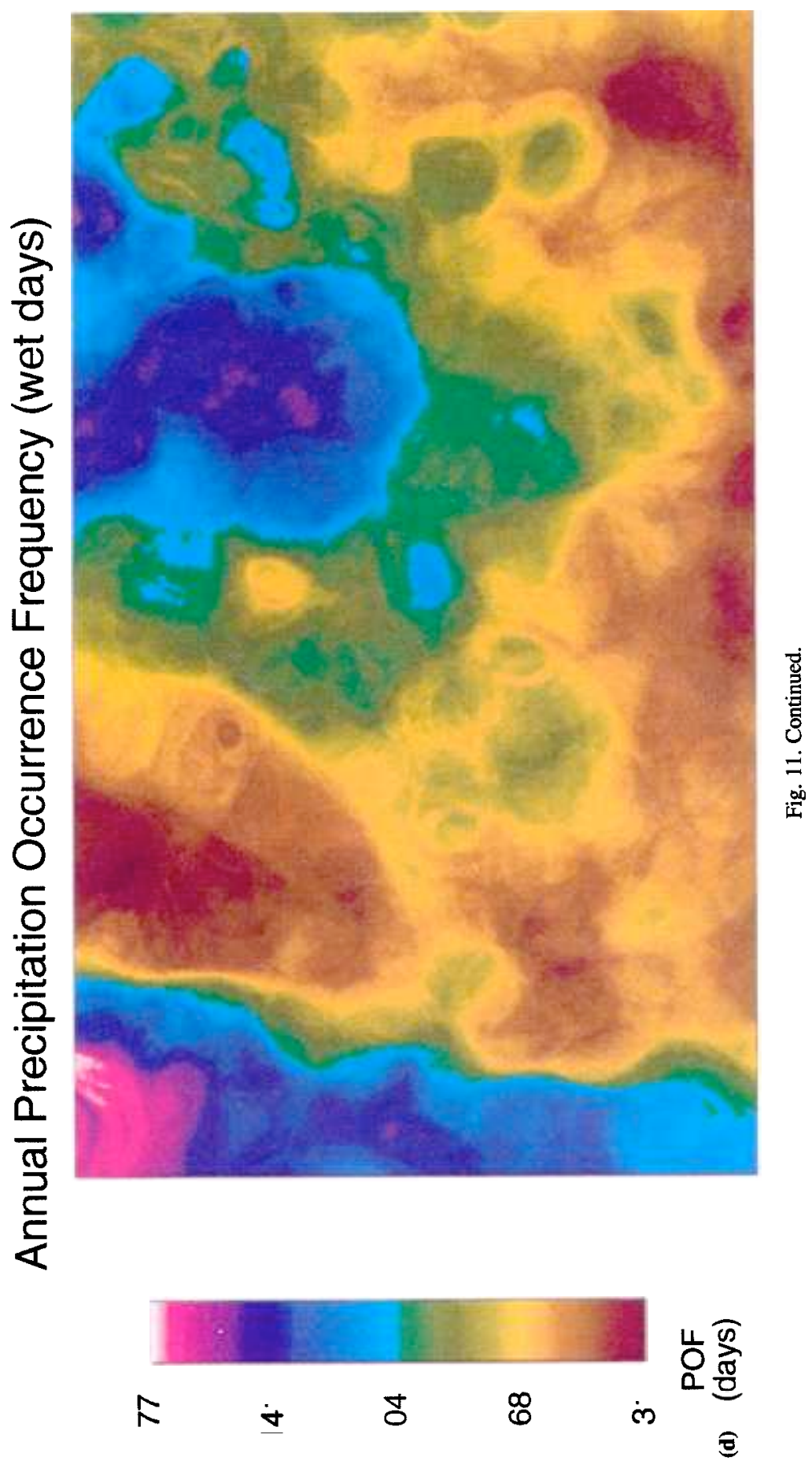

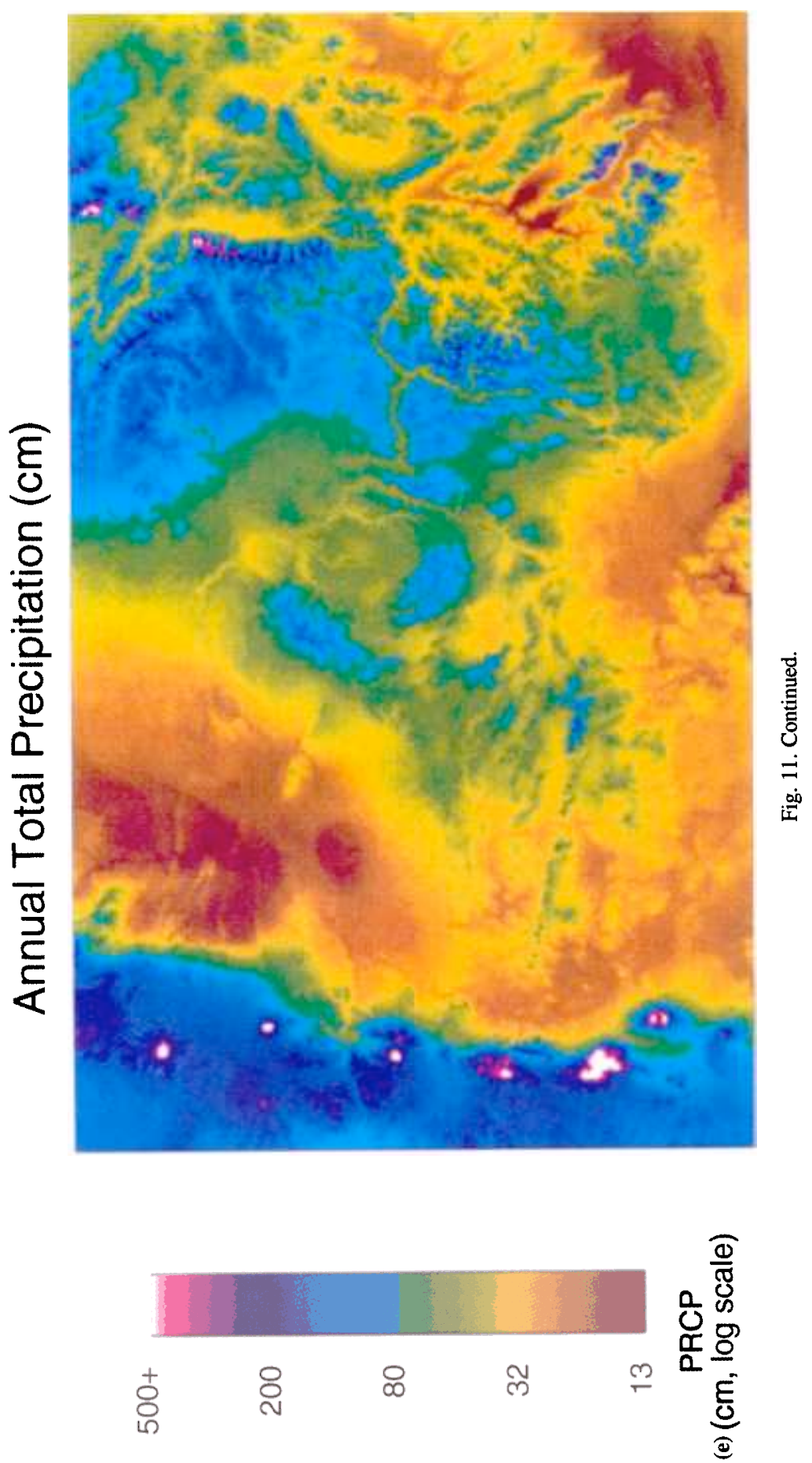


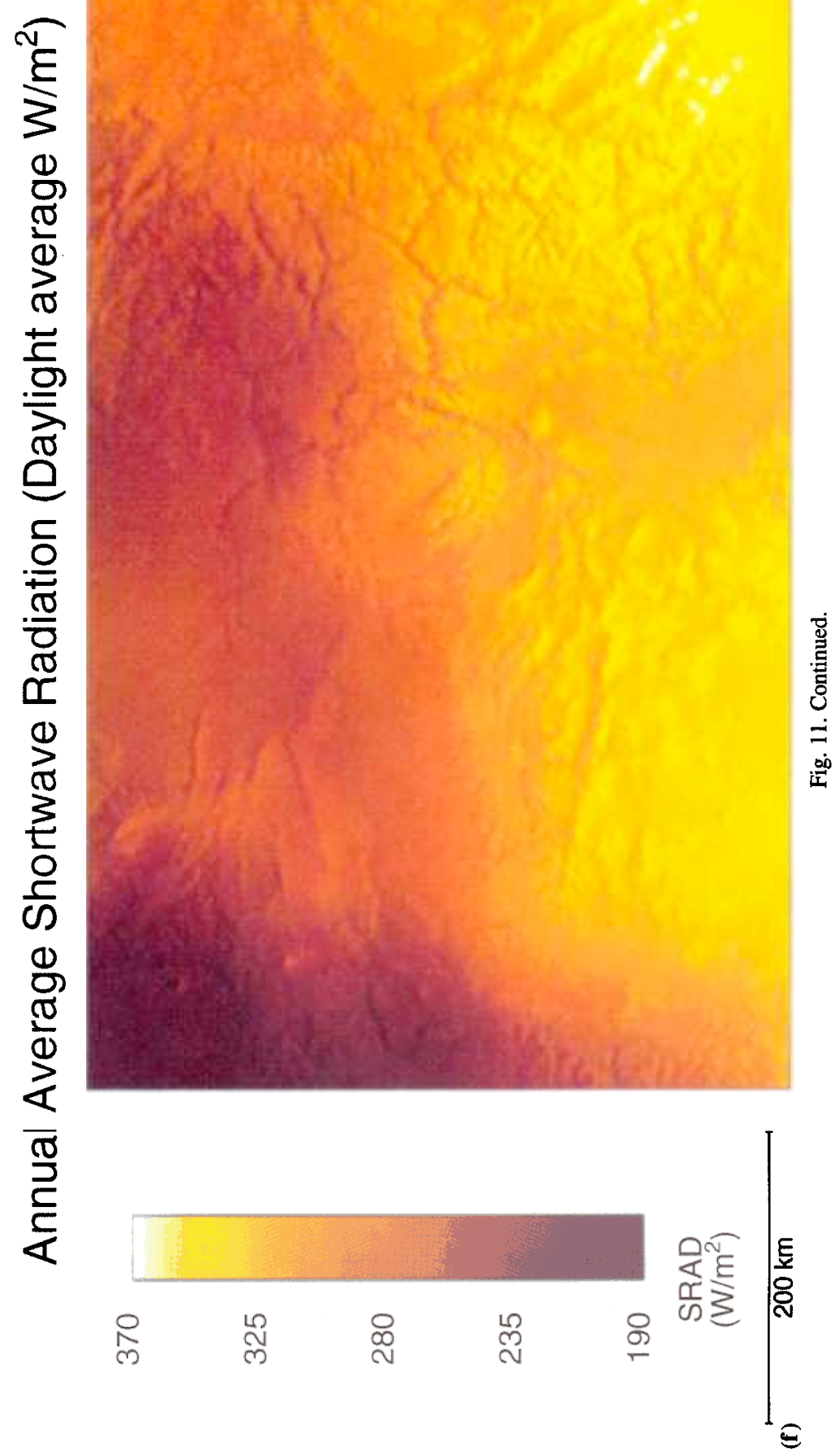


in the application of these methods in a climatological mode, but a more detailed analysis is warranted.

In the future we hope to use the methods described here, in conjunction with remotely sensed observations of surface temperature and cloud cover, in an investigation of largescale parameterizations of surface resistance schemes. Our spatially distributed and relatively accurate near-surface air temperatures, in combination with remotely sensed surface temperature, can provide estimates of surface resistances (Reginato et al., 1985; Seguin et al., 1994). Such estimates, based on observation, can be used to validate and refine spatially explicit estimates of surface resistance that are derived from simulations. In addition, satellite observations of cloud-cover, often seen as a hindrance in remote sensing studies of land surface processes, can be used to test our method's spatially explicit estimation of solar radiation. The combination of surface and satellite observations should allow a regional assessment of evaporation and resultant soil moisture stores as presented by Saha (1995), and could lead to improvements in the initialization of surface moisture fields for coupled atmosphere-terrestrial ecosystem simulations (Pielke et al., 1993). Ten years ago, Eagleson (1986) reported that macroscale field observations were limiting the advance of hydrological science; we see the methods presented here as an attempt to overcome such limitations.

\section{Apknoyledgement}

We express our thanks to Cameron Johnston, Marty Beck, James Menakis, and Robert Keane of the USDA Forest Service Fire Sciences Laboratory for collecting all the station data used here. We also thank Joseph Glassy, John Kimball, Timothy Kittel, Robert Kremer, Ramakrishna Nemani, and Joseph White for helpful discussions. Review comments from Jene Michaud and an anonymous reviewer were instrumental in improving an earlier draft of this manuscript. This study was funded by USDA Forest Service Contract INT94932-RJVA, Cooperative Park Service Project 1268-0-9001, and NASA Grant NAGW-4234. P.E.T. was supported by a NASA Graduate Student Fellowship in Global Change Research.

\section{References}

Band, L.E., Peterson, D.L., Running, S.W., Coughlan, J.C., Lammers, R., Dungan, J. and Nemani, R.R., 1991. Forest ecosystem processes at the watershed scale: basis for distributed simulation. Ecol. Modelling, 56: $171-$ 196.

Band, L.E., Patterson, P., Nemani, R.R. and Running, S.W., 1993. Forest ecosystem processes at the watershed scale: incorporating hillslope hydrology. Agric. For. Meteorol., 63: 93-126.

Bristow, K.L. and Campbell, G.S., 1984. On the relationship between incoming solar radiation and daily maximum and minimum temperature. Agric. For. Meteorol., 31: 159-166.

Chua, S.-H. and Bras, R.L., 1982, Optimal estimators of mean areal precipitation in regions of orographic influence. J. Hydrol., 57: 23-48.

Creutin, J.D. and Obled, C., 1982. Objective analyses and mapping techniques for rainfall fields: an objective comparison. Water Resour. Res., 18: 413-431. 
Daly, C., Neilson, R.P. and Phillips, D.L., 1994. A statistical-topographic model for mapping climatological precipitation over mountainous terrain. J. Appl. Meteorol., 33: 140-158.

DeGaetano, A.T., Eggleston, K.L. and Knapp, W.W., 1995. A method to estimate missing daily maximum and minimum temperature observations. J. Appl. Meteorol., 34: 371-380.

Dolph, J. and Marks, D., 1992. Characterizing the distribution of observed precipitation and runoff over the continental United States. Climatic Change, 22: 99-119.

Eagleson, P.S., 1986. The emergence of global-scale hydrology. Water Resour. Res., 22: 6S-14S.

Forsythe, W.C., Rykiel, E.J., Stahl, R.S., Wu, H. and Schoolfield, R.M., 1995. A model comparison for daylength as a function of latitude and day of year. Ecol. Modelling, 80: 87-95.

Gandin, L.S., 1965. Objective Analysis of Meteorological Fields. Israel Program for Scientific Translations, Jerusalem, 242 pp.

Glassy, J.M. and Running, S.W., 1994. Validating diurnal climatology logic of the MT-CLIM model across a climatic gradient in Oregon. Ecol. Appl., 4: 248-257.

Hayden, C.M. and Purser, R.J., 1995. Recursive filter analysis of meteorological fields: applications to NESDIS operational processing. J. Appl. Meteorol., 34: 3-15.

Hevesi, J.A., Istok, J.D. and Flint, A.L., 1992. Precipitation estimation in mountainous terrain using multivariate geostatistics. Part I: Structural analysis. J. Appl. Meteorol., 31: 661-676.

Hungerford, R.D., Nemani, R.R., Running, S.W. and Coughlan, J.C., 1989. MTCLIM: a mountain microclimate simulation model. US For. Serv. Res. Pap. INT-414.

Hutchinson, M.F., 1995. Stochastic space-time weather models from ground-based data. Agric. For. Meteorol., 73: 237-264.

Hutchinson, M.F. and Bischof, R.J., 1983. A new method for estimating the spatial distribution of mean seasonal and annual rainfall applied to the Hunter Valley, New South Wales. Aust. Meteorol. Mag., 31: 179-184.

Kimball, J., Running, S.W. and Nemani, R.R, 1997. An improved method for estimating surface humidity from daily minimum temperature. Agric. For. Meteorol., in press.

McMurtrie, R.E., Leuning, R., Thompson, W.A. and Wheeler, A.M., 1992. A model of canopy photosynthesis and water use incorporating a mechanistic formulation of leaf $\mathrm{CO}_{2}$ exchange. For. Ecol. Manage., 52: 261278.

Murray, F.W., 1967. On the computation of saturation vapor pressure. J. Appl. Meteorol., 6: 203-204.

Nemani, R.R., Pierce, L., Band, L.E. and Running, S.W., 1993. Forest ecosystem processes at the watershed scale: sensitivity to remotely sensed leaf area index observations. Int. J. Remote Sens., 14: 2519-2534.

Phillips, D.L., Dolph, J. and Marks, D., 1992. A comparison of geostatistical procedures for spatial analysis of precipitation in mountainous terrain. Agric. For. Meteorol., 58: 119-141.

Pielke, R.A., Garstang, M., Lindsey, C. and Gusdorf, J., 1987. Use of a synoptic classification scheme to define systems. Theor. Appl. Climatol., 38: 57-68.

Pielke, R.A., Schimel, D.S., Lee, T.J., Kittel, T.G.F. and Zeng, X., 1993. Atmosphere-terrestrial ecosystem interactions: implications for coupled modeling. Ecol. Modelling, 67: 5-18.

Reginato, R.J., Jackson, R.D. and Pinter, P.J., 1985. Evapotranspiration calculated from remote multispectral and ground station meteorological data. Remote Sens. Environ., 18: 75-89.

Running, S.W., 1994. Testing FOREST-BGC ecosystem process simulations across a climatic gradient in Oregon. Ecol. Appl., 4: 238-247.

Running, S.W. and Coughlan, J.C., 1988. A general model of forest ecosystem processes for regional applications. I. Hydrologic balance, canopy gas exchange, and primary production processes. Ecol. Modelling, 42: $125-154$.

Running, S.W. and Nemani, R.R., 1991. Regional hydrologic and carbon balance responses of forests resulting from potential climate change. Climatic Change, 19:349-368.

Running, S.W., Nemani, R.R. and Hungerford, R.D., 1987. Extrapolation of synoptic meteorological data in mountainous terrain and its use for simulating forest evapotranspiration and photosynthesis. Can. J. For. Res., 17: 472-483.

Saha, S.K., 1995. Assessment of regional soil moisture conditions by coupling satellite sensor data with a soilplant system heat and moisture balance model. Int. J. Remote Sens., 16: 973-980.

Seguin, B., Courault, D. and Guérif, M., 1994. Surface temperature and evapotranspiration: application of local scale methods to regional scales using satellite data. Remote Sens. Environ., 49: 287-295. 
Shuttleworth, W.J., 1988. Macrohydrology—the new challenge for process hydrology. J. Hydrol., 100: 31-56. Tabios, G.Q. and Salas, J.D., 1985. A comparative analysis of techniques for spatial interpolation of precipitation. Water Resour. Bull., 21: 365-380.

Thiessen, A.H., 1911. Precipitation averages for large areas. Mon. Weather Rev., 39: 1082-1084.

Troch, P.A., Mancini, M., Paniconi, C. and Wood, E.F., 1993. Evaluation of a distributed catchment scale water balance model. Water Resour. Res., 29: 1805-1817.

USDA Soil Conservation Service, 1988. Snow Survey and Water Supply Products Reference. West National Technical Center, Snow Survey Program, Portland, Oregon, 12 pp.

Wallis, T.W.R. and Griffiths, J.F., 1995. An assessment of the weather generator (WXGEN) used in the erosion/ productivity impact calculator (EPIC). Agric. For. Meteorol., 73: 115-133.

White, J.D. and Running, S.W., 1994. Testing scale dependent assumptions in regional ecosystem simulations. J. Veg. Sci., 5: 687-702.

Willmott, C.J. and Robeson, S.M., 1995. Climatologically aided interpolation (CAI) of terrestrial air temperature. Int. J. Climatol., 15: 221-229. 